\title{
Deletion of a conserved Gata2 enhancer impairs haemogenic endothelium programming and adult haematopoiesis
}

Tomasz Dobrzycki ${ }^{1}$, Christopher B. Mahony ${ }^{5}$, Monika Krecsmarik ${ }^{1,2}$, Cansu Koyunlar ${ }^{3}$, Rossella Rispoli ${ }^{1,4}$, Joke Peulen-Zink ${ }^{3}$, Kirsten Gussinklo ${ }^{3}$, Bakhta Fedlaoui ${ }^{5}$, Emma de Pater ${ }^{3}$, Roger Patient ${ }^{1,2}$ and Rui Monteiro ${ }^{1,2,5,{ }^{*}}$

1. MRC Molecular Haematology Unit, MRC Weatherall Institute of Molecular Medicine, John Radcliffe Hospital, University of Oxford, Oxford, OX3 9DS, United Kingdom

2. BHF Centre of Research Excellence, Oxford, United Kingdom

3. Department of Hematology, Erasmus MC, Rotterdam, The Netherlands

4. Division of Genetics and Molecular Medicine, NIHR Biomedical Research Centre, Guy's and St Thomas' NHS Foundation Trust and King's College London, London, UK

5. Institute of Cancer and Genomic Sciences, College of Medical and Dental Sciences, University of Birmingham, Birmingham, B15 2TT, United Kingdom

*correspondence:

Email: r.monteiro@bham.ac.uk 


\section{Abstract}

Haematopoietic stem and progenitor cells (HSPCs) maintain the vertebrate blood system throughout life and their emergence from haemogenic endothelium (HE) is regulated by transcription factors such as Gata2. Here we deleted a conserved enhancer (i4 enhancer) driving pan-endothelial expression of gata2a and showed that Gata2a is required for HE programming by regulating expression of runx 1 and of the second zebrafish Gata2 orthologue, gata2b. By 5 days, homozygous gata2a $a^{\Delta i / \Delta i 4}$ larvae showed normal numbers of HSPCs, a recovery mediated by Notch signalling driving gata $2 b$ and run $x 1$ expression in HE. However, gata2 $a^{\Delta i 4 / \Delta i 4}$ adults showed oedema, susceptibility to infections and marrow hypo-cellularity, consistent with bone marrow failure found in GATA2 deficiency syndromes. Thus, gata2a expression driven by the i4 enhancer is required for HE programming in embryos and maintenance of steady-state haematopoietic stem cell output in the adult. These enhancer mutants are a new paradigm to explore the pathophysiology of GATA2-related deficiencies in vivo.

Keywords: gata2a, gata2b, haemogenic endothelium, haematopoietic stem cells, bone marrow failure 


\section{Introduction}

Haematopoietic stem cells (HSCs) are the source of all blood produced throughout the lifetime of an organism. They are capable of self-renewal and differentiation into progenitor cells that generate specialised blood cell types. DNA-binding transcription factors are fundamental players in the inception of the haematopoietic system as it develops in the embryo, but also play a crucial role in maintaining homeostasis of the haematopoietic system in the adult organism. They are part of elaborate gene regulatory networks that coordinate differentiation, proliferation and survival of haematopoietic cells and ensure their levels are appropriate at all times throughout life. The only time during ontogenesis when HSCs are generated de novo is during embryonic development and misexpression of key transcription factors may lead to a failure to produce HSCs or, alternatively, to haematopoietic disorders and eventually leukaemia. Therefore, understanding how transcription factors drive the haematopoietic process provides opportunities for intervention when haematopoiesis is dysregulated.

The development of blood occurs in distinct waves: primitive, pro-definitive and definitive, each of them characterised by the generation of blood progenitors in a specific location and restricted in time, where the definitive wave produces multi-lineage self-renewing $\mathrm{HSCs}^{1}$. The specification of HSCs initiates in cells with arterial characteristics and proceeds through an endothelial intermediate, termed the haemogenic endothelium $(\mathrm{HE})^{2}$. In zebrafish and other vertebrates, expression of runx1 in the floor of the dorsal aorta defines the bona fide HE population ${ }^{3,4}$. Haematopoietic stem and progenitor cells (HSPCs) emerge from the HE by endothelial-to-haematopoietic transition (EHT), both in zebrafish and in mice ${ }^{5-7}$. They first arise at around 34 hours post fertilisation (hpf) from the HE in the ventral wall of the $\mathrm{DA}^{8}$, the analogue of the mammalian $\mathrm{AGM}^{9}$. After EHT, the HSCs enter the bloodstream through the posterior cardinal vein (PCV) ${ }^{8}$ to colonise the caudal haematopoietic tissue $(\mathrm{CHT})$, the zebrafish equivalent of the mammalian foetal liver ${ }^{10}$. Afterwards the HSCs migrate again within the bloodstream to colonise the kidney marrow (WKM) and thymus ${ }^{8}$, the final niche for HSCs, equivalent to the bone marrow in mammals ${ }^{1}$. 
Gata2 is a key haematopoietic transcription factor (TF) in development. In humans, GATA2

haploinsufficiency leads to blood disorders, including MonoMAC syndrome (Monocytopenia, Mycobacterium avium complex) and myeloid dysplastic syndrome (MDS) ${ }^{11,12}$. While its presentation is variable, MonoMAC syndrome patients always show cytopenias, ranging from mild to severe, and hypocellular bone marrow ${ }^{12,13}$. These patients are susceptible to mycobacterial and viral infections and have a propensity to develop myelodysplastic syndrome (MDS) and Acute Myeloid Leukaemia (AML), with a $75 \%$ prevalence and relatively early onset at age $20^{12}$.

Gata2 knockout mice are embryonic lethal and die by E10.5 ${ }^{14}$. Conditional Gata2 knockout under the control of the endothelial VE-cad promoter abolished the generation of intra-aortic clusters ${ }^{15}$, suggesting that Gata2 is required for HSPC formation. In addition, conditional deletion of Gata2 mediated by a Vav-Cre transgene demonstrated that Gata2 is also required for the maintenance and survival of HSCs in the foetal liver, after HE specification and HSC emergence ${ }^{15}$. Further studies in the mouse revealed a decrease in HSC numbers in Gata2 heterozygous mutants, but also a dose-dependency of adult HSCs on Gata2 ${ }^{16}$.

Gata2 expression in the endothelium is regulated by an intronic enhancer element termed the +9.5 enhancer ${ }^{17,18}$. Deletion of this enhancer results in the loss of HSPC emergence from HE, leading to lethality by $\mathrm{E} 14{ }^{18}$. The same element is also mutated in $10 \%$ of all the MonoMAC syndrome patients ${ }^{11}$.

Because of a partial genome duplication during the evolution of teleost fish, numerous zebrafish genes exist in the form of two paralogues, including gata $2^{19}$. This provides an opportunity to separately identify the temporally distinct contributions made by each Gata2 orthologue. Gata2a and gata $2 b$ are only 57\% identical and are thought to have undergone evolutionary sub-functionalisation from the ancestral vertebrate Gata2 gene ${ }^{20,21}$. Gata2b is expressed in HE in the floor of the dorsal aorta from $18 \mathrm{hpf}$ and is required for runx1 expression in $\mathrm{HE}^{20}$. In addition, lineage tracing experiments showed that gata2bexpressing HE cells gave rise to HSCs in the adult ${ }^{20}$. Similar to the mouse Gata2, gata $2 b$ expression depends on Notch signalling and is a bona fide marker of $\mathrm{HE}$, currently regarded as the functional 'haematopoietic homologue' of Gata2 in zebrafish ${ }^{20}$. By contrast, gata2a is expressed in all endothelial cells and in the 
developing central nervous system ${ }^{20,22}$. Homozygous gata $a^{u m 27}$ mutants showed arteriovenous shunts in the dorsal aorta at $48 \mathrm{hpf}^{23}$. However, gata2a is expressed at $11 \mathrm{hpf}$ in the haemangioblast population in the posterior lateral mesoderm (PLM) that gives rise to the arterial endothelial cells in the trunk ${ }^{24}$, well before gata $2 b$ is expressed in HE. This suggests that gata $2 a$ might play a role in endothelial and $\mathrm{HE}$ programming and thus help to elucidate an earlier role for Gata2 in HSC development.

Here we show that the gata2a locus contains a conserved enhancer in its $4^{\text {th }}$ intron, corresponding to the described $+9.5 \mathrm{~Kb}$ enhancer in the mouse Gata2 locus ${ }^{17,18}$, a feature that was not found in the gata2b locus. Using CRISPR/Cas9 genome editing, we demonstrated that this region, termed the i4 enhancer, is required for endothelial-specific gata2a expression. Analysis of homozygous mutants (gata2a $a^{\Delta i / \Delta i 4}$ mutants) showed decreased expression of the HE-specific genes runx1 and gata2b. Thus, endothelial expression of gata2a, regulated by the i4 enhancer, is required for gata $2 b$ and runx 1 expression in the HE. Strikingly, their expression recovers and by $48 \mathrm{hpf}$, the expression of haematopoietic markers in gata $2 a^{\Delta i 4 / \Delta i 4}$ mutants is indistinguishable from wild type siblings. We have demonstrated that this recovery is mediated by an independent input from Notch signalling, sufficient to recover runx1 expression in HE and thus HSPC emergence by $48 \mathrm{hpf}$. We conclude that runx 1 and gata $2 \mathrm{~b}$ are regulated by two different inputs, one Notchindependent input from Gata2a and a second from the Notch pathway, acting as a fail-safe mechanism for the initial specification of HSPCs in the absence of the input by Gata2a. Despite the early rescue, gata $2 a^{\Delta i 4 / \Delta i 4}$ adults showed increased susceptibility to infections, oedema, a hypocellular kidney marrow (WKM) and neutropenia, a phenotype resembling key features of GATA2 deficiency syndromes in humans. We conclude that Gata2a is required to maintain steady-state haematopoietic output from adult HSPCs and this function requires the activity of the i4 enhancer. 


\section{Results}

\section{A region of open chromatin in intron 4 of the zebrafish gata2a locus is specific to endothelium and}

\section{highly conserved}

Because Gata2 genes are duplicated in zebrafish, we set out to unpick the different roles Gata2a and Gata2b play during HSC generation and homeostasis by identifying their regulatory regions. Analysis of sequence conservation revealed that one region within the fourth intron of the zebrafish gata2a locus was conserved in vertebrates, including mouse and human (Fig. 1a-c). This region, which we termed 'i4 enhancer', corresponds to the endothelial +9.5 Gata2 enhancer identified previously in the mouse ${ }^{17,18}$ and human ${ }^{25}$. Notably, the gata $2 b$ locus did not show broad conservation in non-coding regions (Supplementary Fig. 1a).

To investigate whether the i4 element was a potentially active enhancer, we first performed ATAC-seq ${ }^{26}$ to identify open chromatin regions in endothelial cells (ECs) in zebrafish. We used a $\mathrm{Tg}(k d r l: G F P)$ transgenic line that expresses GFP in all endothelium ${ }^{27}$ and isolated the higher GFP-expressing ECs (kdrl:GFPhigh, termed $k d r l: G F P^{+}$for simplicity) as this fraction was enriched for endothelial markers compared to the kdrl:GFPlow fraction (Supplementary Fig. 1b,c). Principal Component Analysis on the ATAC-seq data from 26hpf $k d r l: G F P^{+}$cells $(n=2)$ and $k d r l: G F P^{-}$cells $(n=4)$ revealed strong differences between the open chromatin regions in the two cell populations, further supported by a correlation analysis (Supplementary Fig. $1 \mathrm{~d}$-f). 78,026 peaks were found in common between replicates of the ATACseq in $k d r l: \mathrm{GFP}^{+}$cells (Supplementary Fig. 1g). 44,025 peaks were differentially expressed between the kdrl:GFP ${ }^{+}$and $k d r l: G F P^{-}$ fractions (Supplementary Fig. 1h). An analysis of known motifs present in the $k d r l: G F P^{+}$population revealed an enrichment for the ETS motif (Supplementary Fig. 1i). ETS factors are essential regulators of gene expression in endothelium ${ }^{28}$. In addition, we performed gene ontology (GO) term analysis on the peaks showing $>3$-fold enrichment or depletion in ECs (Supplementary Fig. $1 \mathrm{j}-\mathrm{I}$ ). As expected, non-ECs showed a broad range of GO terms whereas EC-enriched peaks were associated with terms like angiogenesis or blood vessel development (Supplementary Fig. 1k,I). 
Differential peak analysis in the gata2a locus identified four differentially open sites within a $20 \mathrm{~kb}$ genomic region (Fig. 1b), including one peak in intron 4 corresponding to the predicted i4 enhancer. It contained a core $150 \mathrm{bp}$-long element that included several binding motifs for the Gata, E-box and Ets transcription factor families (Fig. 1b). Although the positioning of the E-box site relative to the adjacent GATA site differs in zebrafish and mammals (Fig. 1b,c), the necessary spacer distance of $\sim 9 b p$ between the two sites ${ }^{29}$ was conserved. Thus, this site may be a target for TF complexes containing an E-box-binding factor and a GATA family TF.

Thus, the intronic enhancer (i4) identified in the zebrafish gata2a locus is accessible to transposase in endothelial cells and contains highly conserved binding sites for key haematopoietic transcription factors, suggesting that genetic regulation of gata2a expression in zebrafish $\mathrm{HE}$ is a conserved feature of vertebrate gata2 genes.

The gata2a-i4 enhancer drives GFP expression in the endothelium, including the haemogenic endothelium

To investigate the activity of the gata2a-i4 enhancer in vivo, the conserved genomic $150 \mathrm{bp}$ region (Fig. $1 \mathrm{~b}, \mathrm{c}$ ), together with flanking $\pm 500 \mathrm{bp}$ (gata2a-i4-1.1kb:GFP) or $\pm 150 \mathrm{bp}$ (gata2a-i4-450bp:GFP) was cloned into a Tol2-based reporter E1b:GFP construct ${ }^{30}$ and used to generate stable transgenic lines (Supplementary Fig. 2). The earliest activity of the enhancer was observed at the 14-somite stage (14ss), when gfp mRNA was detected in the PLM (Supplementary Fig. 2a,b). After $22 \mathrm{hpf}$, the reporter signal was pan-endothelial (Fig. 1d-e, Supplementary Fig. 2c-i). Around 27hpf, higher intensities of GFP fluorescence and corresponding higher levels of $g f p$ mRNA were visible in the floor of the DA (Fig. 1d-e, Supplementary Fig. 2e-h). While the GFP protein was still visible in the vasculature around 3dpf, it was likely carried over from earlier stages, since the gfp mRNA was not detectable anymore (Supplementary Fig. 2i,j). We focussed our subsequent analysis on the gata2a-i4-1.1kb:GFP transgenics as they showed stronger expression of the transgene (Supplementary Fig. 2k). At 25hpf, the expression of GFP protein and gfp mRNA overlapped 
completely in the endothelial cells of the DA (Fig. 1f-f"). Overall, these data confirm that the i4 enhancer is active in vivo in endothelial cells at the correct time to regulate definitive haematopoiesis. The endothelial activity of the corresponding +9.5 enhancer was also observed in mouse embryos ${ }^{17}$, indicating functional conservation of the gata2a-i4 enhancer across vertebrates.

To further characterise the enhancer activity in vivo, $\operatorname{Tg}$ (gata2a-i4-1.1kb:GFP) embryos were stained for gata2a mRNA and for GFP protein (Supplementary Fig. 2k-o). We found a large overlap between gata2a ${ }^{+}$ and $\mathrm{GFP}^{+}$cells at $30 \mathrm{hpf}$ in the DA, with a small proportion of $\mathrm{GFP}^{+}$cells that did not express gata2a mRNA ( $<5 \%$, Supplementary Fig. 20). This could suggest that some cells require activity of other endothelial enhancers to trigger transcription of gata2a or that $g f p$ mRNA has a longer half-life than gata2a mRNA. Importantly, the GFP signal was absent in gata2a-expressing neural cells (Supplementary Fig. 2I-n), indicating that the i4 enhancer is specifically active in (haemogenic) endothelial cells.

Next we examined the expression of the HE marker runx $1^{3}$ in gata $2 a-i 4-1.1 \mathrm{~kb}$ :GFP embryos at $25 \mathrm{hpf}$. At this stage, over $90 \%$ of $r u n x 1^{+}$cells were GFP ${ }^{+}$(Fig. 1g-h). We conclude that the GFP expression under the gata2a-i4 enhancer marks the majority of the HE population.

\section{Deletion of the gata2a-i4 enhancer results in decreased expression of gata2a in the embryonic endothelium without affecting endothelial development}

To investigate whether endothelial-specific expression of gata2a is required for definitive haematopoiesis, we deleted the conserved gata2a-i4 enhancer using CRISPR/Cas9 genome editing ${ }^{31}$. We generated a deletion mutant lacking $231 \mathrm{bp}$ of the i4 enhancer (Supplementary Fig. 3a-c) and named it gata $2 a^{\Delta \mathrm{ii} / \Delta \mathrm{\Delta i} 4}$. Homozygous gata2 $a^{\Delta i 4 / \Delta i 4}$ mutants showed decreased levels of gata2a expression in endothelial cells when compared to wild type embryos (Fig. 2a,b). By contrast, gata2a expression in the neural tube appeared unaffected in the gata2 $a^{\mathrm{\Delta i} / / \Delta \mathrm{i}}$ mutants (Fig. 2a,b). At 28hpf, expression of the pan-endothelial marker kdrl was indistinguishable between wild type and gata $2 a^{\Delta \mathrm{ii} / \Delta \mathrm{i} 4}$ mutants (Fig. $2 \mathrm{c}, \mathrm{d}$ ). To verify these results, we crossed homozygous gata2 $a^{\Delta i 4 / \Delta i 4}$ mutants to $\mathrm{Tg}(k d r l: G F P)$ transgenics and analysed vascular morphology. 
Gata2a $a^{\Delta i / \Delta i 4}$ embryos showed no gross vascular abnormalities at $48 \mathrm{hpf}$ as assessed by the expression of the $\operatorname{Tg}(k d r l: G F P)$ transgene (Fig. 2e,f).

Next, we isolated endothelial cells from $\operatorname{Tg}(k d r l: G F P)$ and $\operatorname{Tg}(k d r l: G F P) ; ~ g a t a 2 a^{\Delta i 4 / \Delta i 4}$ embryos by FACS (Fig.

$2 \mathrm{~g}$ ) at $23 \mathrm{hpf}$ and $30 \mathrm{hpf}$ and confirmed by qRT-PCR that the endothelial markers $k d r l$, $d l d$ and $d l l 4$ were unaffected in gata2 $a^{\Delta i / / \Delta i 4}$ embryos (Supplementary Fig. 3d-f). The arterial marker efnb2a ${ }^{32}$ was decreased at $23 \mathrm{hpf}$ in gata2 $a^{\Delta \mathrm{i} / / \Delta \mathrm{i} 4}$ mutants but recovered by $30 \mathrm{hpf}$ (Supplementary Fig. 3g). In addition, gata2a was significantly decreased in the $k d r l: G F P^{+} E C s$ in $23 \mathrm{hpf}$ gata2a $a^{\Delta i 4 / \Delta i 4}$ embryos compared to wild types (Fig. $2 \mathrm{~h}$ ). At $30 \mathrm{hpf}$ this decrease was not statistically significant. This was likely due to a decrease in expression of gata2a that appears to occur in wild type ECs during development, whereas gata2a expression in mutants remained low (Fig. 2h). Importantly, there was no difference in gata2a expression in the non-endothelial population ( $k d r l: G F P^{-}$cells) between wild type and gata $2 a^{\Delta i 4 / \Delta i 4}$ mutants at either $23 \mathrm{hpf}$ or $30 \mathrm{hpf}$ (Fig. $2 \mathrm{i}$ ). Altogether, these data suggest that genomic deletion of the gata2a-i4 enhancer is sufficient to reduce expression of gata2a specifically in endothelium.

Deletion of the gata2a-i4 enhancer results in reduced expression of runx 1 and gata $2 b$ at early stages of HE specification

To investigate a potential role of gata2a in HSC development, we compared the expression of runx1, the key marker of $\mathrm{HE}$ in zebrafish ${ }^{3}$, in wild type and gata2 $a^{\Delta i 4 / \Delta i 4}$ embryos. Quantitative in situ hybridization (ISH) analysis ${ }^{33}$ showed that runx1 expression was decreased in gata2a $a^{\Delta i 4 / \Delta i 4}$ embryos at $24 \mathrm{hpf}$ (Supplementary Fig. 4a-c) and 28hpf compared to wild type siblings (Fig. 3a-c) . Further analysis in kdrl:GFP ECs showed that this decrease in runx 1 expression was already detectable at $23 \mathrm{hpf}$ in gata $2 a^{\Delta \mathrm{ii} / \Delta \mathrm{i} 4}$ mutants (Fig. 3d), at the onset of its expression in $\mathrm{HE}^{34}$. Thus, deletion of the gata2a-i4 enhancer results in impaired runx1 expression in the early stages of HE programming. This correlates well with decreased runx1 expression levels in $+9.5^{-1-}$ mouse AGM explants ${ }^{18}$, further supporting the critical evolutionary role of the intronic enhancer of Gata2 in HSC specification. 
Next, we tested whether Gata2a could act upstream of gata $2 b$ by measuring gata $2 b$ expression in gata2a $a^{\Delta i / / \Delta i 4}$ embryos. Quantitation of the ISH signal showed that gata2 $b$ expression was decreased in gata2a $a^{\Delta i / / \Delta i 4}$ embryos compared to wild type siblings at 26hpf (Supplementary Fig. 4d) and 28hpf (Fig. 3e-g), but recovered to wild type levels by $30 \mathrm{hpf}$ (Supplementary Fig. 4e). Accordingly, $k d r l: \mathrm{GFP}^{+} ;$gata2a $a^{\Delta i 4 / \Delta i 4}$ cells express significantly lower levels of gata $2 b$ mRNA than the wild type $k d r l: G F P^{+}$endothelial population in $23 \mathrm{hpf}$ embryos, but not at $30 \mathrm{hpf}$ (Fig. 3h). These data suggest that endothelial expression of gata2a is required upstream of gata $2 b$ and runx 1 for the proper specification of $\mathrm{HE}$, uncovering a previously unrecognized role for Gata2a in definitive haematopoiesis.

\section{Expression of late markers of embryonic HSC activity is unaffected in gata $2 a^{\Delta i 4 / \Delta i 4}$ mutants}

The qPCR analysis in sorted $k d r l: \mathrm{GFP}^{+} ;$gata2 $a^{\Delta \mathrm{\Delta i} / \Delta \mathrm{\Delta i}}$ and wild type $k d r l: G F P^{+} \mathrm{ECs}$ (Fig. $3 \mathrm{~d}$ ) already suggested a recovery of of runx1 expression from 30hpf. Of note, the $k d r l: \mathrm{GFP}^{+}$population likely includes the $k d r l^{+}$, runx1-expressing EMPs located in the caudal region ${ }^{35}$. This region was not included in the quantification of $I S H$ but cannot be separated by sorting for $k d r l: \mathrm{GFP}^{+}$and could thus explain the discrepancy between image quantification and qRT-PCR. To further characterize the haematopoietic phenotype in the gata2a $a^{\Delta i 4 / \Delta i 4}$ mutants, we tested whether expression of markers of haematopoietic activity in the embryo was affected from 48hpf onwards (Fig. 4).

At $48 \mathrm{hpf}$, the expression of runx 1 in the DA showed no significant difference between gata $2 a^{\Delta i / / \Delta i 4}$ mutants and wild type controls (Fig. 4a,b). These data suggest that the decrease of runx1 expression at early stages of HE programming in gata $2 a^{\Delta i 4 / \Delta i 4}$ mutants is transient and recovers by $2 \mathrm{dpf}$. Indeed, analysis of the HSPC marker $c m y b^{10}$ in the $\mathrm{CHT}$ at $4 \mathrm{dpf}$ showed no differences between gata2 $2 a^{\Delta \mathrm{i} / / \Delta \mathrm{i} 4}$ and wild type larvae (Fig. 4c,d). Expression of the T-cell progenitor marker rag1 in the thymus ${ }^{36}$ showed that around half of the gata2a $a^{\Delta i / / \Delta i 4}$ larvae had reduced rag1 expression at $4 \mathrm{dpf}$ compared to wild type (Fig. 4e,f). This effect was absent at $5 \mathrm{dpf}$ (Fig. 4g, h), suggesting that HSPC activity was normal in gata2 $2 a^{\Delta \mathrm{i} / / \Delta \mathrm{i} 4}$ mutants from $4 \mathrm{dpf}$ onwards. Next, we crossed the gata2 $a^{\Delta i 4 / \Delta i 4}$ mutants to $\operatorname{Tg}(i t g a 2 b$ :GFP) transgenics, where itga2b-GFP high 
and itga $2 b$-GFPlow cells in the CHT mark thrombocytes and HSPCs, respectively ${ }^{8,37}$. Our analysis revealed no difference in itga $2 b$-GFPlow HSPC or itga2b-GFPhigh thrombocyte numbers in the CHT region at $5 \mathrm{dpf}$ between wild type and gata2a $a^{\Delta i / / \Delta i 4}$ mutants (Fig.4i-I). Taken together, our data suggest that endothelial gata2a expression mediated by the i 4 enhancer is required for the initial expression of gata $2 b$ and $r u n \times 1$ in the HE but largely dispensable after $2 \mathrm{dpf}$.

\section{Haematopoietic recovery of gata $2 a^{\Delta i 4 / \Delta i 4}$ mutants is mediated by the Notch-gata $2 b$ pathway}

The recovery of gata $2 b$ expression by $30 \mathrm{hpf}$ (Fig. 3h, Supplementary Fig. 4e) coincides temporally with the observed decrease in gata2a in wild type endothelial cells (Fig. 2h). Thus, we reasoned that other regulators of gata $2 b$ might compensate for the lack of endothelial gata $2 a$ in gata $2 a^{\Delta i 4 / \Delta i 4}$ mutants and thus lead to a recovery of the initial haematopoietic phenotype. Therefore, we investigated whether the loss of gata $2 b$ in gata $2 a^{\Delta \mathrm{i} / / \Delta \mathrm{i}}$ background resulted in a more severe haematopoietic phenotype than observed in the gata $2 a^{\Delta i 4 / \Delta i 4}$ mutants. For this, we injected gata $2 a^{\Delta i 4 / \Delta i 4}$ and wild type controls with a sub-optimal amount (7.5ng) of a gata $2 b$ morpholino oligonucleotide (MO) ${ }^{20}$. Quantitative ISH analysis confirmed that this amount of gata2b MO had no effect on runx1 expression at 32hpf (Fig. 5a,b). As expected, runx1 expression in gata2a $a^{\Delta i / \Delta \Delta / 4}$ embryos was significantly reduced compared to wild type siblings (Fig. 5a,b). Gata $2 b$ knockdown in gata $2 a^{\Delta i / / \Delta i 4}$ embryos further reduced runx1 expression (Fig. 5a,b). To test whether this stronger reduction of runx 1 at $32 \mathrm{hpf}$ affected later stages of embryonic haematopoiesis, we assessed cmyb expression in the CHT at 4dpf (Fig. 5c). We scored cmyb expression levels as 'wild type' or 'reduced' and found that the 'reduced' embryos were largely overrepresented in the gata2 $a^{\Delta i 4 / \Delta i 4}$ mutants injected with the gata2b MO, compared to wild type fish and non-injected gata2 $a^{\Delta \mathrm{i4} / \Delta i 4}$ siblings (Fig. 5c).

To verify whether Gata2b is required for definitive haematopoiesis downstream of Gata2a, we generated a frameshift truncating mutant for Gata $2 \mathrm{~b}^{38}$ and incrossed gata $2 a^{\Delta i 4 /+} ;$ gata $2 b^{+/-}$adults to investigate $c m y b$ expression at 33hpf in their progeny. Gata $2 b^{-/}$mutants showed a more severe decrease in cmyb expression than gata $2 a^{\Delta \mathrm{i} 4 / \Delta \mathrm{\Delta} 4}$ mutants (Supplementary Fig. 4f-i). Double gata $2 b^{-1} ;$ gata2 $2 a^{\Delta i / / \Delta i 4}$ mutants showed no 
further reduction in cmyb expression compared to gata $2 b^{-/-}$mutants, suggesting that Gata2a was not sufficient to drive cmyb expression in HE in the absence of Gata2b (Supplementary Fig. 4f-i). Taken together, we conclude that Gata2b is regulated by Gata2a and is required for definitive haematopoiesis.

Next, we tested whether forced ectopic expression of gata $2 b$ was sufficient to speed up the haematopoietic recovery of gata2 $a^{\Delta i 4 / \Delta i 4}$ embryos. Thus, we overexpressed gata2b under the control of the gata2a-i4-450bp enhancer in wild type and gata2a $a^{\Delta \mathrm{i4} / \Delta 4}$ mutant embryos and measured runx1 expression at 28hpf in the DA. Gata2a $a^{\Delta i / \Delta i 4}$ embryos showed a significant decrease in runx1 expression in compared to wild type (Fig. 5d). Ectopic expression of gata $2 b$ under the gata2a-i4 enhancer significantly increased runx 1 expression in wild type and mutants (Fig. 5d). Importantly, it was sufficient to bring the runx1 expression levels in the mutants up to the levels detected in uninjected wild type embryos (Fig. $5 \mathrm{~d}$ ), suggesting that gata $2 b$ alone was sufficient to drive runx1 expression in the ventral wall of the DA and drive the haematopoietic recovery in gata $2 a^{\Delta i 4 / \Delta i 4}$ mutants. Thus, gata $2 b$ can recover the definitive haematopoietic programme in the absence of endothelial gata2a.

Because the expression of gata $2 b$ is regulated by Notch signalling ${ }^{20}$, we investigated whether inhibition of Notch would also prevent the haematopoietic recovery of gata2 $a^{\Delta i 4 / \Delta i 4}$ embryos. For this, we used the Notch inhibitor DAPM ${ }^{39}$, and titrated it down to a sub-optimal dose $(25 \mu \mathrm{M})$ that did not significantly affect runx1 expression (Supplementary Fig. 5a). This dose induced a small but measurable decrease in gata2b expression in DAPM-treated embryos while higher doses had a more robust effect (Supplementary Fig. 5b). Next, we treated wild type and gata $2 a^{\Delta i / / \Delta i 4}$ mutant embryos with DAPM and measured runx1 expression in the DA at 36hpf (Fig. 5e,f). Suboptimal DAPM treatment did not affect runx1 expression in wild type embryos (Fig. 5e,f), but gata2 $2 a^{\Delta i 4 / \Delta i 4}$ mutants showed lower runx1 levels and DAPM treatment further reduced runx 1 expression (Fig. 5e,f). By contrast, gata $2 b$ expression at $36 \mathrm{hpf}$ was unaffected by $25 \mu \mathrm{M}$ DAPM but strongly reduced by $100 \mu \mathrm{M}$ DAPM (Supplementary Fig. 5c). Taken together, these data show that Notch activity is sufficient to drive haematopoietic recovery in gata $2 a^{\Delta \mathrm{\Delta i} / \Delta i 4}$ mutants. Consistent with these results, ectopic activation of Notch signalling in endothelium with a fli1a-NICD:GFP construct ${ }^{40}$ led to 
increased runx1 and gata2b expression in wild type embryos at 30hpf (Supplementary Fig. 5d,e). When overexpressed in gata2 $a^{\Delta i 4 / \Delta i 4}$ mutants, fli1a-NICD:GFP rescued runx1 expression to near wild type levels at 26hpf (Supplementary Fig. 5f). Thus, we conclude that HE programming requires two independent inputs on runx1 and gata2b expression; one from Gata2a, driven in ECs by the i4 enhancer, and the other from Notch signalling, necessary and sufficient to drive HE programming even in the absence of gata2a.

\section{Adult gata $2 a^{\Delta \mathrm{i} 4 / \Delta \mathrm{i} 4}$ mutants show defects resembling GATA2 haploinsufficiency}

To investigate whether Gata2a plays a role in adult haematopoiesis, we first asked whether the gata2a-i41.1kb:GFP reporter was active in haematopoietic cells in the adult. Whole kidney marrow (WKM) cells isolated from the transgenic fish showed that the i4 enhancer is active in haematopoietic cells previously defined by flow cytometry ${ }^{41}$ as progenitors, lymphoid+HSPC (containing the HSPCs) and myeloid cells (Supplementary Fig. 6a-c). Accordingly, single cell transcriptional profiling showed higher levels of gata2a in HSPCs, progenitors, neutrophils and thrombocytes (Supplementary Fig. 6d-f) ${ }^{42,43}$. Consistent with this notion, we observed a high incidence of infections and heart oedemas in gata $2 a^{\Delta \mathrm{ii} / \Delta \mathrm{i} 4}$ adult fish, with over $25 \%$ suffering from one of these defects by 6 months of age, compared to $<1 \%$ of wild type fish (Fig. 6a-c). The heart oedemas and the infections are suggestive of lymphatic defects and immune deficiency as observed in human patients bearing genetic GATA2 haploinsufficiency syndromes such as MonoMAC syndrome ${ }^{12}$. Notably, around $10 \%$ of MonoMAC syndrome patients show mutations in the homologous enhancer region of GATA2 ${ }^{11,13}$.

Next, we counted the total number of haematopoietic cells in wild type and gata $2 a^{\Delta \mathrm{i} / / \Delta \mathrm{i} 4}$ mutant WKM (Fig. $6 \mathrm{~d}-\mathrm{f})$. To avoid any confounding effects in our analysis, we compared wild type to gata2 $2 a^{\Delta i / / \Delta i 4}$ mutants without overt signs of infection. The gata $2 a^{\Delta i 4 / \Delta i 4}$ mutants showed a $\sim 2$-fold decrease in the total number of haematopoietic cells in the WKM (Fig. 6d-f). In addition, neutrophils were similarly reduced (Fig. 6g), another characteristic in common with MonoMAC syndrome patients ${ }^{13}$. Lastly, kidney marrow smears of ten 9-month old gata $2 a^{\Delta i 4 / \Delta i 4}$ mutants were assessed. One of the ten mutants showed an excess of 
immature myeloid blast cells in the WKM (>98\%) and only minor erythrocyte differentiation (Fig. 6h,i). The presence of excess blasts is usually an indication of AML in humans. Together these data strongly suggest that the i4 enhancer is a critical driver of gata2a expression in adult haematopoietic cells. The enhancer deletion in gata $2 a^{\Delta \mathrm{\Delta i} / \Delta i 4}$ mutants leads to a hypocellular WKM and neutropenia, strongly suggestive of marrow failure, a hallmark of disease progression in Gata2 deficiency syndromes.

\section{Discussion}

\section{Endothelial expression of gata2a through a conserved intronic enhancer is required for HE programming}

The sub-functionalisation of the Gata2 paralogues in zebrafish provided an opportunity to unpick the different roles of Gata2 in the multi-step process of definitive haematopoiesis. Here we have investigated the conservation of the Gata2 +9.5 enhancer and identified a homologous region in intron 4 of the zebrafish gata2a locus (gata2a-i4) that is not present in the gata $2 b$ locus. The zebrafish gata2a-i4 enhancer, like the mouse enhancer ${ }^{17}$, is sufficient to drive pan-endothelial expression of GFP and necessary for endothelial expression of gata2a (Fig. 1,2). We traced the activity of the i4 enhancer back to the PLM, the source of precursors of endothelium and $\mathrm{HSCs}^{9}$. This degree of sequence and functional conservation of the i4 enhancer led us to hypothesize that Gata2a might play a role in definitive haematopoiesis. Indeed, homozygous deletion of the i4 enhancer (gata2 $a^{\Delta i 4 / \Delta i 4}$ ) allowed us to uncover a previously unknown function of Gata2a in regulating the initial expression of runx1 and gata $2 b$ in HE. Although $c m y b$ expression in $\mathrm{HE}$ was decreased in gata $2 a^{\Delta \mathrm{i} / \Delta \mathrm{\Delta i4}}$ mutants, it was more severely reduced in gata $2 b^{-/}$mutants, suggesting that Gata2b is more important for cmyb regulation than Gata2a. However, both Gata2 orthologues regulate gene expression in the HE before the first reported EHT events at $34 \mathrm{hpf}^{5}$.

\section{Regulation of gata2a and gata2b}


Gata2 binds to the +9.5 enhancer to maintain its own expression in endothelial and haematopoietic cells ${ }^{25,44}$. In zebrafish, it is likely that Gata2a binds the GATA motifs in the i4 enhancer and loss of gata2a in the endothelium of gata2a $a^{\Delta i / \Delta / 4}$ mutants (Fig. 2) seems to support this view. Interestingly, we detected a small region in intron 4 of the gata $2 b$ locus that was not identified as a peak in our ATACseq experiment but is conserved in some fish species (Supplementary Fig. 1a) and thus could potentially represent a divergent gata $2 \mathrm{~b}$ intronic enhancer. We speculate that the positive autoregulation of Gata2 was likely retained by both gata2 orthologues in zebrafish. In this case, Gata2a would bind to the gata2b intronic enhancer in HE only until enough Gata2b is present, at which point its intronic enhancer would 'switch' Gata2a for Gata2b to maintain gata2b expression. This 'switch', however, would replace one activator for another, rather than replacing an activating Gata factor (Gata2) for a repressive Gata factor as described previously for the Gata2/Gata1 switch in erythroid cells ${ }^{45}$. Alternatively, the positive auto-regulation function might have been modified in HE so that Gata2a binds to both gata2a and gata2b intronic enhancers to regulate their expression. These possibilities remain to be investigated.

\section{Rescue of the haematopoietic defects in gata2 $a^{\Delta \mathrm{ii} / \Delta \mathrm{\Delta i}}$ mutants}

The gata $2 a^{\Delta i 4 / \Delta i 4}$ mutants recovered from the early defects in HE programming and displayed normal expression levels of cmyb in the $\mathrm{CHT}$ at $4 \mathrm{dpf}$ and rag1 in the thymus at $5 \mathrm{dpf}$, used as indicators of the definitive haematopoietic programme ${ }^{10}$. We hypothesized that this could be due to the presence of the two homologues of Gata2 in zebrafish ${ }^{19}$, despite Gata2a and Gata2b proteins being only $50 \%$ identical ${ }^{20}$. Indeed, forced expression of gata $2 b$ under the gata $2 a-i 4$ enhancer rescued the expression of runx 1 in the gata $2 a^{\Delta i 4 / \Delta i 4}$ mutants to wild type levels and sub-optimal depletion of gata $2 b$ in the gata $2 a^{\Delta i 4 / \Delta i 4}$ mutants resulted in more severe reduction in cmyb expression in the CHT by $4 \mathrm{dpf}$ (Fig.4). In addition, we demonstrated that Notch signalling, a known regulator of gata $2 b$ expression ${ }^{20}$, is sufficient to rescue the initial HE programming defect induced by deletion of the gata2a-i4 enhancer. We propose a model in which gata2a acts upstream of runx 1 and gata $2 b$ independently of Notch to initiate HE programming. The regulation of gata $2 b$ by Gata2a is transient, and the timing largely coincides with the natural decrease in 
endothelial expression of gata2a by $30 \mathrm{hpf}$. After this stage, endothelial Notch signalling takes over the regulation of $r u n \times 1$ and gata $2 b$ expression, acting as a fail-safe mechanism that buffers against fluctuations in the system caused by loss of one or more of the initial inputs (in this case, Gata2a).

In the mouse, $+9.5^{-\%}$ enhancer mutants exhibit decreased haematopoietic output from cultured AGM explants ${ }^{18}$ or foetal liver $\mathrm{HSCs}^{25}$. In both cases the experiments were performed at E11.5, well after the HE had been established and given rise to $\mathrm{HSCs}^{4}$. At E11.5 the numbers of haematopoietic cells in the AGM were roughly comparable to their wild type siblings ${ }^{18,25}$, raising the possibility that an initial HE defect might have gone unnoticed in those studies. Thus, by analysing the contribution of two zebrafish Gata2 paralogues, we uncovered a previously unappreciated contribution by a Gata2 gene in the programming of HE prior to HSC specification.

\section{Loss of i4 enhancer activity leads to a phenotype resembling human GATA2 deficiency syndromes}

Despite the apparent haematopoietic recovery, we observed a high incidence of infections and oedema in gata $2 a^{\Delta i 4 / \Delta i 4}$ adults, and a striking decrease in the number of haematopoietic cells in the WKM. The decrease in haematopoietic cells in particular is reminiscent of the loss of proliferative potential of haematopoietic Gata2 ${ }^{+/-}$heterozygous cells in the mouse ${ }^{16,46}$. This raises the possibility that in zebrafish the gata2a and gata $2 b$ paralogues may function as two Gata2 'alleles' that together regulate the haematopoietic output of the WKM. This will be addressed by comparing the adult phenotypes of gata $2 a^{\Delta i 4 / \Delta i 4}$ and gata $2 b^{-/-}$mutants.

Taken together, our initial characterization of WKM shows that gata2 $a^{\Delta i / / \Delta i 4}$ mutants present a phenotype consistent with Gata2 deficiency syndromes in humans brought about by GATA2 haploinsufficiency ${ }^{11,25}$. Strikingly, about $10 \%$ of all MonoMAC patients show mutations in the conserved +9.5 enhancer ${ }^{11,13}$, the corresponding regulatory element to the i 4 enhancer. The i4 enhancer is active in the lymphoid+HSPC fraction that contains the HSC activity ${ }^{47}$, in the progenitor cells and in the myeloid fraction that contains eosinophils, previously identified as expressing high levels of a gata2a-GFP BAC transgenic reporter ${ }^{41}$. 
bioRxiv preprint doi: https://doi.org/10.1101/516203; this version posted December 10, 2019. The copyright holder for this preprint (which was not certified by peer review) is the author/funder, who has granted bioRxiv a license to display the preprint in perpetuity. It is made available under aCC-BY-NC-ND 4.0 International license.

Thus, it is likely that gata $2 a^{\Delta i / / \Delta i 4}$ adult fish show lineage-specific differentiation defects. Further characterization of the gata $2 a^{\Delta i / / \Delta i 4}$ mutants will uncover which haematopoietic cells are most affected by the loss of i 4 enhancer activity and how Gata2a regulates haematopoietic output, thus establishing a novel animal model for human diseases linked to Gata2 haploinsufficiency. 


\section{Methods}

\section{Maintenance of zebrafish}

Zebrafish (Danio rerio) were maintained in flowing system water at $28.5^{\circ} \mathrm{C}$, conductance $450-550 \mu \mathrm{S}$ and $\mathrm{pH}$ $7.0 \pm 0.5$ as described ${ }^{48}$. Fish suffering from infections or heart oedemas were culled according to Schedule 1 of the Animals (Scientific Procedures) Act 1986. Eggs were collected by natural mating. Embryos were grown at $24-32^{\circ} \mathrm{C}$ in $\mathrm{E} 3$ medium with methylene blue and staged according to morphological features (Kimmel et al. 1995) corresponding to respective age in hours or days post fertilization (hpf or dpf, respectively). Published lines used in this work were wild type (wt $\left.{ }^{\mathrm{KCL}}\right), \mathrm{Tg}\left(-6.0\right.$ itga2 $^{2}$ :EGFP) ${ }^{\mathrm{Ia} 237,49}$ and $\mathrm{Tg}(k d r l: G F P)^{8843} 27$. All animal experiments were approved by the relevant University of Oxford, University of Birmingham and Erasmus University ethics committees.

\section{ATAC-seq}

$\mathrm{Tg}(k d r l: G F P)^{5843}$ embryos were dissociated for FACS at 26-27hpf to collect $k d r l^{+}$and $k d r l^{-}$cell populations (40,000-50,000 cells each). They were processed for ATAC library preparation using optimised standard protocol ${ }^{26}$. Briefly, after sorting into Hanks' solution (1xHBSS, $0.25 \% \mathrm{BSA}, 10 \mathrm{mM}$ HEPES pH8), the cells were spun down at $500 \mathrm{~g}$ at $4^{\circ} \mathrm{C}$, washed with ice-cold PBS and resuspended in $50 \mu \mathrm{l}$ cold Lysis Buffer $(10 \mathrm{mM}$ Tris$\mathrm{HCl}, 10 \mathrm{mM} \mathrm{NaCl}, 3 \mathrm{mM} \mathrm{MgCl}_{2}, 0.1 \%$ IGEPAL, $\left.\mathrm{pH} 7.4\right)$. The nuclei were pelleted for $10 \mathrm{~min}$. at $500 \mathrm{~g}$ at $4^{\circ} \mathrm{C}$ and resuspended in the TD Buffer with Tn5 Transposase (Illumina), scaling the amounts of reagents accordingly to the number of sorted cells. The transposition reaction lasted $30 \mathrm{~min}$. at $37^{\circ} \mathrm{C}$. The DNA was purified with PCR Purification MinElute Kit (QIAGEN). In parallel, transposase-untreated genomic DNA from $k d r l^{+}$cells was purified with the DNeasy ${ }^{\circledR}$ Blood \& Tissue Kit (QIAGEN). The samples were amplified with appropriate Customized Nextera primers ${ }^{26}$ in NEBNext High-Fidelity 2x PCR Master Mix (NEB). The libraries were purified with PCR Purification MinElute Kit (QIAGEN) and Agencourt AMPure XP beads (Beckmann Coulter). The quality of each library was verified using D1000 ScreenTape System (Agilent). Four biological replicas of the libraries were quantified with the KAPA Library Quantification Kit for Illumina ${ }^{\circ}$ platforms (KAPA 
Biosystems). The libraries were pooled (including the Tn5-untreated control), diluted to $1 \mathrm{ng} / \mu \mathrm{l}$ and sequenced using 75bp paired-end reads on Illumina HiSeq 4000 (Wellcome Trust Centre for Human Genetics, Oxford). Raw sequenced reads were checked for base qualities, trimmed where $20 \%$ of the bases were below quality score 20, and filtered to exclude adapters using Trimmomatic (Version 0.32) and mapped to Zv9 reference genome (comprising 14,612 genes) ${ }^{50}$ using BWA with default parameters. The results were visualised using UCSC Genome Browser (http://genome-euro.ucsc.edu/) ${ }^{51}$. The eight data sets were analysed with Principal Component Analysis (PCA) to identify outliers. Correlation among kdrl:GFP+ and $k d r l: G F P$ - samples was assessed with a tree map. The peaks were called for each sample using the Tn5-untreated control as input. We identified the common peaks between replicates and then used DiffBind (EdgeR method) to identify differential peaks between $k d r l: G F P^{+}$and $k d r l: G F P$ 'samples (Supplementary Table 2). The threshold for differential peaks was $p<0.05$.

Generation of transgenic Tg(gata2a-i4-1.1kb:GFP), Tg(gata2a-i4-450bp:GFP) and mutant gata2a $a^{\Delta i 4 / \Delta i 4}$ and $g a t a 2 b^{-1-}$ zebrafish lines Genomic regions containing the identified $150 \mathrm{bp}$-long gata2a-i4 enhancer flanked by $\pm 500 \mathrm{bp}$ (i4-1.1kb) or $\pm 150 \mathrm{bp}$ (i4-450bp) were amplified from wild type zebrafish genomic DNA with NEB Phusion polymerase (see Supplementary Table 1 for primer sequences) and cloned upstream of E1b minimal promoter and GFP into a Tol2 recombination vector (Addgene plasmid \#37845, ${ }^{52}$ ) with Gateway cloning technology (Life Technologies $^{\mathrm{TM}}$ ) following the manufacturer's protocol. One-cell zebrafish embryos were injected with $1 \mathrm{nl}$ of an injection mix, containing 50pg gata2a-i4-E1b-GFP-Tol2 construct DNA + 30pg tol2 transposase mRNA 30. Transgenic founders (Tg(gata2a-i4-1.1kb:GFP) and (gata2a-i4-450bp:GFP)) were selected under a widefield fluorescent microscope and outbred to wt fish. Carriers of monoallelic insertions were detected by the Mendelian distribution of $50 \%$ fluorescent offspring coming from wt outcrosses. These transgenics were then inbred to homozygosity.

To generate the i4 deletion mutant, we identified potential sgRNA target sites flanking the 150bp conserved region within intron 4 of the gata2a locus (see Fig. 1A, Fig S3A). sgRNAs were designed with the 
CRISPR design tool (http:/crispr.mit.edu/, see Supplementary Table 1 for sequences) and prepared as described ${ }^{31}$. To reduce potential off-target effects of CRISPR/Cas9, we utilized the D10A 'nickase' version of Cas9 nuclease ${ }^{53,54}$, together with two pairs of sgRNAs flanking the enhancer (Supplementary Table 1, Supplementary Fig. 3A-B). We isolated two mutant alleles with deletions of $215 \mathrm{bp}(\Delta 78-292)$ and $231 \mathrm{bp}$ ( $\Delta 73-303)$ (Supplementary Fig. 3B). Both deletions included the highly conserved E-box, Ets and GATA transcription factor binding sites (Supplementary Fig. 3B). The $\Delta 73-303$ allele was selected for further experiments and named $\Delta \mathrm{i} 4$. Adult zebrafish were viable and fertile as heterozygous (gata2 $a^{\Delta i 4 /+}$ ) or homozygous (gata2a $a^{\Delta i 4 / \Delta i 4}$ ). To unambiguously genotype wild types, heterozygotes and homozygous mutants, we designed a strategy consisting of two PCR primer pairs (Supplementary Fig. 3A, C). One primer pair flanked the whole region, producing a 600bp wild type band and 369bp mutant band. In the second primer pair, one of the primers was designed to bind within the deleted region, only giving a 367bp band in the presence of the wild type allele (Supplementary Fig. 3C).

To generate the gata $2 \mathrm{~b}$ mutant we designed a CRISPR/Cas9 strategy for a frameshift truncating mutant in exon 3 deleting both zinc fingers. sgRNAs were designed as described above and guides were prepared according to Gagnon et al. ${ }^{55}$ with minor adjustments. Guide RNAs were generated using the Agilent SureGuide gRNA Synthesis Kit, Cat\# 5190-7706. Cas9 protein (IDT) and guide were allowed to form ribonucleoprotein structures (RNPs) at RT and injected in 1 cell stage oocytes. 8 embryos were selected at $24 \mathrm{hpf}$ and lysed for DNA isolation. Heteroduplex PCR analysis was performed to test guide functionality and the other embryos from the injection were allowed to grow up. To aid future genotyping we selected mutants by screening F1 for a PCR detectable integration or deletion in exon 3. Sequence verification showed that founder 3 had a 28 nt integration resulting in a frameshift truncating mutation leading to 3 new STOP codons in the third exon. To get rid of additional mutations caused by potential off target effects, founder 3 was crossed to WT for at least 3 generations. All experiments were performed with offspring of founder 3. 


\section{Fluorescence-activated cell sorting (FACS)}

$\sim 100$ embryos at the required stage were collected in Low Binding ${ }^{\circ}$ SafeSeal ${ }^{\circ}$ Microcentrifuge Tubes

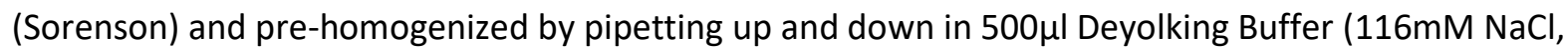
$2.9 \mathrm{mM} \mathrm{KCl}, 5 \mathrm{mM}$ HEPES, $1 \mathrm{mM}$ EDTA). They were spun down for $1 \mathrm{~min}$. at $500 \mathrm{~g}$ and incubated for $15 \mathrm{~min}$. at $30^{\circ} \mathrm{C}$ in Trypsin + Collagenase Solution (1xHBSS, $0.05 \%$ Gibco $^{\circ}$ Trypsin+EDTA (Life Technologies ${ }^{\mathrm{Tm}}$ ), 20mg/ml collagenase (Sigma)). During that time, they were homogenized by pipetting up and down every $3 \mathrm{~min}$. The lysis was stopped by adding $50 \mu$ l foetal bovine serum and $650 \mu$ l filter-sterilized Hanks' solution (1xHBSS,

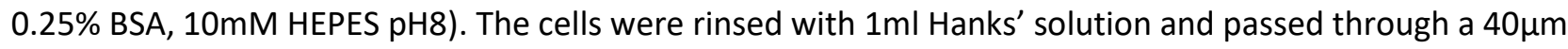
cell strainer (Falcon ${ }^{\circ}$ ). They were resuspended in $\sim 400 \mu$ l Hanks' solution with 1:10,000 Hoechst 33258 (Molecular Probes ${ }^{\circ}$ ) and transferred to a $5 \mathrm{ml}$ polystyrene round bottom tube for FACS sorting. The cells were sorted on FACSAria Fusion sorter by Kevin Clark (MRC WIMM FACS Facility). The gates of GFP (488530) and DsRed (561-582) channels were set with reference to samples derived from non-transgenic embryos. The fluorescence readouts were compensated when necessary. For ATAC-seq library preparation, the cells were sorted into Hank's solution. For RNA isolation, the cells were sorted directly into RLT Plus buffer (QIAGEN) + 1\% $\beta$-mercaptoethanol and processed with the RNEasy ${ }^{\circ}$ Micro Plus kit (QIAGEN), according to the accompanying protocol. The RNA was quantified and its quality assessed with the use of Agilent RNA 6000 Pico kit. All RNA samples were stored at $-80^{\circ} \mathrm{C}$.

\section{SYBR $^{\circ}$ Green qRT-PCR}

$3 \mu \mathrm{l}$ of the cDNA diluted in $\mathrm{H}_{2} \mathrm{O}$ were used for technical triplicate qRT-PCR reactions of $20 \mu \mathrm{l}$ containing the Fast SYBR ${ }^{\circ}$ Green Master Mix (Thermo Fisher Scientific) and appropriate primer pair (see Supplementary Table 1). The reactions were run on 7500 Fast Real-Time PCR System (Applied Biosystems) and the results were analysed with the accompanying software. No-template controls were run on each plate for each primer pair. Each reaction was validated with the melt curve analysis. The baseline values were calculated automatically for each reaction. The threshold values were manually set to be equal for all the reactions 
run on one plate, within the linear phase of exponential amplification. The relative mRNA levels in each sample were calculated by subtracting the geometric mean of $\mathrm{Ct}$ values for housekeeping genes eef1a1/1 and $u b c$ from the average $\mathrm{Ct}$ values of the technical triplicates for each gene of interest. This value ( $\Delta \mathrm{Ct})$ was then converted to a ratio relative to the housekeeping genes with the formula $2^{-\Delta C t}$.

\section{Fluidigm Biomark qRT-PCR}

To quantify the differences in gata2a expression between wild type and mutant ECs, we crossed homozygous gata2a $a^{\Delta i / / \Delta i 4}$ mutants to $\mathrm{Tg}(k d r l: G F P)$ transgenics to generate $\mathrm{Tg}(k d r l: G F P) ; ~ g a t a 2 a^{\Delta i 4 / \Delta i 4}$ embryos. These fish, along with non-mutant $\operatorname{Tg}(k d r l: G F P)$, were used for FACS-mediated isolation of $k d r l: \mathrm{GFP}^{+}$and $k d r l: \mathrm{GFP}^{-}$cells to quantitatively compare mRNA expression levels of gata2a in the endothelial and non-endothelial cells of wild type and gata2a $a^{\Delta i 4 / \Delta i 4}$ embryos, using the Fluidigm Biomark ${ }^{\mathrm{TM}}$ qRT-PCR platform. Briefly, 1ng RNA from FACS-sorted cells was used for Specific Target Amplification in a 10 $\mu$ l reaction with the following reagents: $5 \mu \mathrm{l} 2$ XBuffer and $1.2 \mu \mathrm{l}$ enzyme mix from SuperScript III One-Step Kit (Thermo Fisher Scientific), $0.1 \mu \mathrm{l}$ SUPERase $\mathrm{In}^{\mathrm{TM}}$ RNase Inhibitor (Ambion), $1.2 \mu \mathrm{l}$ TE buffer (Invitrogen),

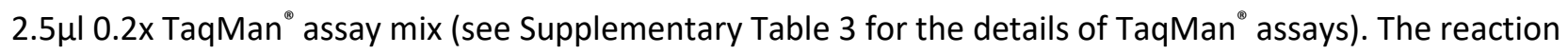
was incubated for $15 \mathrm{~min}$. at $50^{\circ} \mathrm{C}$, for $2 \mathrm{~min}$. at $95^{\circ} \mathrm{C}$ and amplified for 20 cycles of $15 \mathrm{~s}$ at $95^{\circ} \mathrm{C} / 4 \mathrm{~min}$. at $60^{\circ} \mathrm{C}$. The cDNA was diluted 1:5 in TE buffer and stored at $-20^{\circ} \mathrm{C}$. Diluted CDNA was used for qRT-PCR according to the Fluidigm protocol for Gene Expression with the 48.48 IFC Using Standard TaqMan ${ }^{\circ}$ Assays (Table S3). Each sample was run in 3-4 biological replicates. The collected data were analysed with Fluidigm Real-Time PCR Analysis software (version 4.1.3). The baseline was automatically corrected using the built-in Linear Baseline Correction. The thresholds were manually adjusted for each gene to fall within the linear phase of exponential amplification, after which they were set to equal values for the housekeeping genes: $r p / p 0, r p / 13 a, \operatorname{cops} 2^{56}, I s m 12 b^{57}$ and eef1a1/1. The relative mRNA levels for each sample were calculated by subtracting the geometric mean of $\mathrm{Ct}$ values for the housekeeping genes from the $\mathrm{Ct}$ value for each gene of interest. This value $(\Delta \mathrm{Ct})$ was then converted to a ratio relative to the housekeeping genes with the formula $2^{-\Delta C t}$. The $\Delta C$ t values were analysed with 2 -tailed paired-samples $t$-tests with $95 \%$ confidence levels. 
For Fluidigm Biomark ${ }^{\mathrm{TM}}$ qRT-PCR, the $\Delta \mathrm{Ct}$ values were analysed with 2-tailed independent-samples $t$-tests with $95 \%$ confidence levels, using IBM $^{\circ}$ SPSS $^{\circ}$ Statistics (version 22) software.

\section{Flow cytometry and isolation of WKM haematopoietic cells}

Single cell suspensions of WKM cells were prepared from adult zebrafish kidneys of the required genotypes as described ${ }^{58}$. Flow cytometry analysis was performed on a FACS Aria II (BD Biosciences) after exclusion of dead cells by uptake of Hoechst dye (Hoechst 33342, H3570, ThermoScientific), as described ${ }^{41}$. WKM cell counts were performed on a PENTRA ES60 (Hariba Medical) following the manufacturer's instructions. Note that the cell counter does not recognize the zebrafish nucleated erythrocytes, so these were excluded from this analysis. Cell counts for each genotype were analysed with 2-tailed paired-samples $t$-tests with $95 \%$ confidence levels, using a Mann-Whitney test for non-parametric distribution. The scatter plots were generated using GraphPad Prism 8.0 and show medians \pm SD.

\section{May-Grunwald and Wright-Giemsa staining}

Cell staining with May-Grunwald (MG) stain (Sigma MG500) and Giemsa (GIEMSA STAIN, FLUKA 48900) was performed on haematopoietic cell samples. After cytospin, slides are allowed to air-dry and were stained for $5 \mathrm{~min}$ at room temperature with a 1:1 mix of MG:distilled water. Next, slides were drained and stained with a 1:9 dilution of Giemsa:distilled water solution for $30 \mathrm{~min}$ at room temperature. Excess solution was drained and removed by further washes in distilled water. Finally, the slides were air-dried and mounted in DPX (06522, Sigma) for imaging.

Whole mount in situ hybridization (ISH) and immunohistochemistry 
Whole-mount ISH was carried out as described previously ${ }^{59}$, using probes for $k d r l$, runx 1, cmyb, gata2a , gata $2 b, \operatorname{rag} 13,36,60,61$, and gfp (Supplementary Table 1). For conventional ISH embryos were processed, imaged and the ISH signal quantified as described ${ }^{33}$. Briefly, the pixel intensity values were assessed for normal distribution with a Q-Q plot and transformed when necessary. Mean values $(\mu)$ of each experimental group were analysed with 2-tailed independent-samples $t$-tests or with ANOVA with 95\% confidence levels, testing for the equality of variances with a Levene's or Brown-Forsythe test and applying the Welch correction when necessary. For ANOVA, differences between each two groups were assessed with either Tukey's post-hoc test (for equal variances) or with Games-Howell test (for unequal variances). For all these analyses, the IBM ${ }^{\circ}$ SPSS $^{\circ}$ Statistics (version 22) or GraphPad Prism 8.0 package were used. For the analysis of cmyb expression in the $\mathrm{CHT}$ at $4 \mathrm{dpf}$, the embryos scored as 'high' or 'low' were tested for equal distribution between morphants and uninjected controls or among wild type, heterozygous and mutant genotypes with contingency Chi-squared tests, applying Continuity Correction for $2 \times 2$ tables, using $\mathrm{IBM}^{\circ}$ SPSS $^{\circ}$ Statistics (version 22).

For fluorescent ISH (FISH) combined with immunohistochemistry, ISH was performed first following the general whole mount in situ hybridisation protocol. The signal was developed with SIGMAFAST Fast Red TR/Naphthol, the embryos rinsed in phosphate-buffered saline with tween20 (PBT) and directly processed for immunohistochemistry. Embryos were blocked in blocking buffer ( $5 \%$ goat serum/0.3 \% Triton X-100 in PBT) for 1 hour at RT before incubated with primary antibody against GFP (rabbit, 1:500, Molecular Probes), diluted in blocking buffer overnight at $4^{\circ} \mathrm{C}$. Secondary antibody raised in goat coupled to AlexaFluor488 (Invitrogen) was used in 1:500 dilutions for $3 \mathrm{~h}$ at RT. Hoechst 33342 was used as a nuclear counterstain.

Fluorescent images were taken on a Zeiss LSM880 confocal microscope using 40x or 63x oil immersion objectives. Images were processed using the ZEN software (Zeiss). 


\section{Fluorescence microscopy and counting of itga2b-GFPhigh and itga2b-GFPlow cells}

For widefield fluorescence microscopy, live embryos were anaesthetised with $160 \mu \mathrm{g} / \mathrm{ml}$ MS222 and mounted in 3\% methylcellulose and imaged on a AxioLumar V.12 stereomicroscope (Zeiss) equipped with a Zeiss AxioCam MrM. To count itga2b-GFPhigh and itga2b-GFPlow cells in the $\mathrm{CHT}$, $\operatorname{Tg}($ itga2b:GFP; $k d r l: m C h e r r y) ; ~ g a t a 2 a^{\Delta i 4 /+}$ animals were incrossed and grown in E3 medium supplemented with PTU to prevent pigment formation. At $5 \mathrm{dpf}$, the larvae were anaesthetised with MS222 and the tail was cut and fixed for $1 \mathrm{~h}$ at room temperature in 4\% PFA. Next, the tails were mounted on $35 \mathrm{~mm}$ glass bottomed dishes (MAtTEK) in $1 \%$ low melt agarose and imaged using a 40x oil objective on an LSM880 confocal microscope (Zeiss). Cells in the CHT region were counted manually on Z-stacks as 'itga2b:GFPlow'

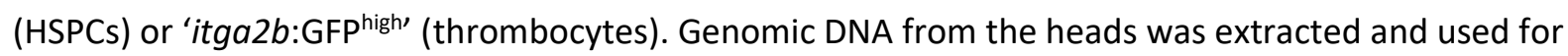
genotyping as described above. Cell counts for each genotype were analysed with 2-tailed paired-samples $t$-tests with 95\% confidence levels, using a Mann-Whitney test for non-parametric distribution. The graphs were generated using GraphPad Prism 8.0 and show medians \pm SD.

\section{References}

1 Ciau-Uitz, A., Monteiro, R., Kirmizitas, A. \& Patient, R. Developmental hematopoiesis: ontogeny, genetic programming and conservation. Exp Hematol 42, 669-683, doi:10.1016/j.exphem.2014.06.001 (2014).

2 Gritz, E. \& Hirschi, K. K. Specification and function of hemogenic endothelium during embryogenesis. Cell Mol Life Sci 73, 1547-1567, doi:10.1007/s00018-016-2134-0 (2016).

3 Kalev-Zylinska, M. L. et al. Runx1 is required for zebrafish blood and vessel development and expression of a human RUNX1-CBF2T1 transgene advances a model for studies of leukemogenesis. Development 129, 2015-2030 (2002).

4 Swiers, G. et al. Early dynamic fate changes in haemogenic endothelium characterized at the singlecell level. Nat Commun 4, 2924, doi:10.1038/ncomms3924 (2013).

5 Kissa, K. \& Herbomel, P. Blood stem cells emerge from aortic endothelium by a novel type of cell transition. Nature 464, 112-115, doi:10.1038/nature08761 (2010).

6 Bertrand, J. Y. et al. Haematopoietic stem cells derive directly from aortic endothelium during development. Nature 464, 108-111, doi:10.1038/nature08738 (2010).

7 Boisset, J. C. et al. In vivo imaging of haematopoietic cells emerging from the mouse aortic endothelium. Nature 464, 116-120, doi:10.1038/nature08764 (2010).

8 Kissa, K. et al. Live imaging of emerging hematopoietic stem cells and early thymus colonization. Blood 111, 1147-1156, doi:10.1182/blood-2007-07-099499 (2008).

9 Davidson, A. J. \& Zon, L. I. The 'definitive' (and 'primitive') guide to zebrafish hematopoiesis. Oncogene 23, 7233-7246, doi:10.1038/sj.onc.1207943 (2004). 
Murayama, E. et al. Tracing hematopoietic precursor migration to successive hematopoietic organs during zebrafish development. Immunity 25, 963-975, doi:10.1016/j.immuni.2006.10.015 (2006). Hsu, A. P. et al. GATA2 haploinsufficiency caused by mutations in a conserved intronic element leads to MonoMAC syndrome. Blood 121, 3830-3837, S3831-3837, doi:10.1182/blood-2012-08452763 (2013).

12 Wlodarski, M. W., Collin, M. \& Horwitz, M. S. GATA2 deficiency and related myeloid neoplasms. Semin Hematol 54, 81-86, doi:10.1053/j.seminhematol.2017.05.002 (2017). Wlodarski, M. W. et al. Prevalence, clinical characteristics, and prognosis of GATA2-related myelodysplastic syndromes in children and adolescents. Blood 127, 1387-1397; quiz 1518, doi:10.1182/blood-2015-09-669937 (2016).

14 Tsai, F. Y. et al. An early haematopoietic defect in mice lacking the transcription factor GATA-2. Nature 371, 221-226, doi:10.1038/371221a0 (1994).

15 de Pater, E. et al. Gata2 is required for HSC generation and survival. J Exp Med 210, 2843-2850, doi:10.1084/jem.20130751 (2013).

16 Ling, K. W. et al. GATA-2 plays two functionally distinct roles during the ontogeny of hematopoietic stem cells. J Exp Med 200, 871-882, doi:10.1084/jem.20031556 (2004).

17 Khandekar, M. et al. A Gata2 intronic enhancer confers its pan-endothelia-specific regulation. Development 134, 1703-1712, doi:10.1242/dev.001297 (2007).

18 Gao, X. et al. Gata2 cis-element is required for hematopoietic stem cell generation in the mammalian embryo. J Exp Med 210, 2833-2842, doi:10.1084/jem.20130733 (2013). Gillis, W. Q., St John, J., Bowerman, B. \& Schneider, S. Q. Whole genome duplications and expansion of the vertebrate GATA transcription factor gene family. BMC Evol Biol 9, 207, doi:10.1186/1471-2148-9-207 (2009).

20 Butko, E. et al. Gata2b is a restricted early regulator of hemogenic endothelium in the zebrafish embryo. Development 142, 1050-1061, doi:10.1242/dev.119180 (2015).

21 Liu, J., Jiang, J., Wang, Z., He, Y. \& Zhang, Q. Origin and evolution of GATA2a and GATA2b in teleosts: insights from tongue sole, Cynoglossus semilaevis. PeerJ 4, e1790, doi:10.7717/peerj.1790 (2016).

22 Yang, L., Rastegar, S. \& Strahle, U. Regulatory interactions specifying Kolmer-Agduhr interneurons. Development 137, 2713-2722, doi:10.1242/dev.048470 (2010).

23 Zhu, C. et al. Evaluation and application of modularly assembled zinc-finger nucleases in zebrafish. Development 138, 4555-4564, doi:10.1242/dev.066779 (2011).

24 Patterson, L. J. et al. The transcription factors Scl and Lmo2 act together during development of the hemangioblast in zebrafish. Blood 109, 2389-2398, doi:10.1182/blood-2006-02-003087 (2007). Johnson, K. D. et al. Cis-element mutated in GATA2-dependent immunodeficiency governs hematopoiesis and vascular integrity. J Clin Invest 122, 3692-3704, doi:10.1172/JCI61623 (2012). Buenrostro, J. D., Giresi, P. G., Zaba, L. C., Chang, H. Y. \& Greenleaf, W. J. Transposition of native chromatin for fast and sensitive epigenomic profiling of open chromatin, DNA-binding proteins and nucleosome position. Nat Methods 10, 1213-1218, doi:10.1038/nmeth.2688 (2013). vascular tube and lumen formation in zebrafish. Development 132, 5199-5209, doi:10.1242/dev.02087 (2005). transcription factors. Semin Cell Dev Biol 22, 976-984, doi:10.1016/j.semcdb.2011.09.009 (2011). Wozniak, R. J. et al. Molecular hallmarks of endogenous chromatin complexes containing master regulators of hematopoiesis. Mol Cell Biol 28, 6681-6694, doi:10.1128/MCB.01061-08 (2008). Kawakami, K. et al. A transposon-mediated gene trap approach identifies developmentally regulated genes in zebrafish. Dev Cell 7, 133-144, doi:10.1016/j.devcel.2004.06.005 (2004).

31 Bassett, A. R., Tibbit, C., Ponting, C. P. \& Liu, J. L. Highly efficient targeted mutagenesis of Drosophila with the CRISPR/Cas9 system. Cell Rep 4, 220-228, doi:10.1016/j.celrep.2013.06.020 (2013). 
32 Lawson, N. D. et al. Notch signaling is required for arterial-venous differentiation during embryonic vascular development. Development 128, 3675-3683 (2001).

33 Dobrzycki, T., Krecsmarik, M., Bonkhofer, F., Patient, R. \& Monteiro, R. An optimised pipeline for parallel image-based quantification of gene expression and genotyping after in situ hybridisation. Biol Open 7, bio031096, doi:10.1242/bio.031096 (2018).

34 Wilkinson, R. N. et al. Hedgehog and Bmp polarize hematopoietic stem cell emergence in the zebrafish dorsal aorta. Dev Cell 16, 909-916, doi:10.1016/j.devcel.2009.04.014 (2009).

35 Bertrand, J. Y. et al. Definitive hematopoiesis initiates through a committed erythromyeloid progenitor in the zebrafish embryo. Development 134, 4147-4156, doi:10.1242/dev.012385 (2007). Willett, C. E., Zapata, A. G., Hopkins, N. \& Steiner, L. A. Expression of zebrafish rag genes during early development identifies the thymus. Dev Biol 182, 331-341, doi:10.1006/dbio.1996.8446 (1997).

37 Lin, H.-F. F. et al. Analysis of thrombocyte development in CD41-GFP transgenic zebrafish. Blood 106, 3803-3810, doi:10.1182/blood-2005-01-0179 (2005).

38 Gioacchino, E. et al. Single cell transcriptome analysis reveals an essential role for Gata2b in hematopoietic lineage decisions in zebrafish. bioRxiv, 753178, doi:10.1101/753178 (2019). Walsh, D. M. et al. Naturally secreted oligomers of amyloid beta protein potently inhibit hippocampal long-term potentiation in vivo. Nature 416, 535-539, doi:10.1038/416535a (2002). Liu, Z. et al. Primary cilia regulate hematopoietic stem and progenitor cell specification through Notch signaling in zebrafish. Nat Commun 10, 1839, doi:10.1038/s41467-019-09403-7 (2019). Traver, D. et al. Transplantation and in vivo imaging of multilineage engraftment in zebrafish bloodless mutants. Nat Immunol 4, 1238-1246, doi:10.1038/ni1007 (2003).

42 Athanasiadis, E. I. et al. Single-cell RNA-sequencing uncovers transcriptional states and fate decisions in haematopoiesis. Nature Communications 8, 2045, doi:10.1038/s41467-017-02305-6 (2017).

43 Macaulay, I. C. et al. Single-Cell RNA-Sequencing Reveals a Continuous Spectrum of Differentiation in Hematopoietic Cells. Cell Rep 14, 966-977, doi:10.1016/j.celrep.2015.12.082 (2016).

44 Sanalkumar, R. et al. Mechanism governing a stem cell-generating cis-regulatory element. Proc Natl Acad Sci U S A 111, E1091-1100, doi:10.1073/pnas.1400065111 (2014).

45 Grass, J. A. et al. Distinct functions of dispersed GATA factor complexes at an endogenous gene locus. Mol Cell Biol 26, 7056-7067, doi:10.1128/MCB.01033-06 (2006).

46 Rodrigues, N. P. et al. Haploinsufficiency of GATA-2 perturbs adult hematopoietic stem-cell homeostasis. Blood 106, 477-484, doi:10.1182/blood-2004-08-2989 (2005).

47 Ma, D., Zhang, J., Lin, H.-f., Italiano, J. \& Handin, R. I. The identification and characterization of zebrafish hematopoietic stem cells. Blood 118, 289-297, doi:10.1182/blood-2010-12-327403 (2011).

48 Westerfield, M. The zebrafish book. A guide for the laboratory use of zebrafish (Danio rerio). 5th edn, (Univ of Oregon Press, 2007).

49 Chi, N. C. et al. Foxn4 directly regulates tbx2b expression and atrioventricular canal formation. Genes Dev 22, 734-739, doi:10.1101/gad.1629408 (2008).

50 Howe, K. et al. The zebrafish reference genome sequence and its relationship to the human genome. Nature 496, 498-503, doi:10.1038/nature12111 (2013).

51 Kent, W. J. et al. The human genome browser at UCSC. Genome Res 12, 996-1006, doi:10.1101/gr.229102 (2002).

52 Birnbaum, R. Y. et al. Coding exons function as tissue-specific enhancers of nearby genes. Genome Res 22, 1059-1068, doi:10.1101/gr.133546.111 (2012).

53 Ran, F. A. et al. Double nicking by RNA-guided CRISPR Cas9 for enhanced genome editing specificity. Cell 154, 1380-1389, doi:10.1016/j.cell.2013.08.021 (2013).

54 Mali, P. et al. CAS9 transcriptional activators for target specificity screening and paired nickases for cooperative genome engineering. Nat Biotechnol 31, 833-838, doi:10.1038/nbt.2675 (2013). 
55 Gagnon, J. A. et al. Efficient mutagenesis by Cas9 protein-mediated oligonucleotide insertion and large-scale assessment of single-guide RNAs. PLoS One 9, e98186, doi:10.1371/journal.pone.0098186 (2014).

$56 \mathrm{Xu}, \mathrm{H}$. et al. Genome-wide identification of suitable zebrafish Danio rerio reference genes for normalization of gene expression data by RT-qPCR. J Fish Bio/ 88, 2095-2110, doi:10.1111/jfb.12915 (2016).

57 Hu, Y., Xie, S. \& Yao, J. Identification of Novel Reference Genes Suitable for qRT-PCR Normalization with Respect to the Zebrafish Developmental Stage. PLoS One 11, e0149277, doi:10.1371/journal.pone.0149277 (2016).

58 Stachura, D. L. \& Traver, D. Cellular dissection of zebrafish hematopoiesis. Methods Cell Biol 133, 11-53, doi:10.1016/bs.mcb.2016.03.022 (2016).

59 Jowett, T. \& Yan, Y. L. Double fluorescent in situ hybridization to zebrafish embryos. Trends Genet 12, 387-389 (1996).

60 Thompson, M. A. et al. The cloche and spadetail genes differentially affect hematopoiesis and vasculogenesis. Dev Biol 197, 248-269, doi:10.1006/dbio.1998.8887 (1998).

61 Monteiro, R. et al. Transforming Growth Factor beta Drives Hemogenic Endothelium Programming and the Transition to Hematopoietic Stem Cells. Dev Cell 38, 358-370,

doi:10.1016/j.devcel.2016.06.024 (2016). 


\section{Acknowledgements}

We thank the staff of the Biomedical Services Units (Oxford, Birmingham and Rotterdam) for fish husbandry. We thank Kevin Clark and Sally-Ann Clarke from the WIMM flow cytometry facility for cell sorting. The flow cytometry facility is supported by the MRC HIU, MRC MHU (MC_UU_12009), NIHR Oxford BRC and John Fell Fund (131/030 and 101/517), the EPA fund (CF182 and CF170), and WIMM Strategic Alliance awards G0902418 and MC_UU_12025. We thank the Wolfson Imaging Centre Oxford imaging. The Wolfson Imaging Centre Oxford is supported by the MRC via the WIMM Strategic Alliance (G0902418), the Molecular Haematology Unit (MC_UU_12009), the Human Immunology Unit (MC_UU_12010), the Wolfson Foundation (grant 18272), and an MRC/BBSRC/EPSRC grant (MR/K015777X/1) to MICA - Nanoscopy Oxford (NanO): Novel Super-resolution Imaging Applied to Biomedical Sciences, Micron (107457/Z/15Z). The facility was supported by WIMM Strategic Alliance awards G0902418 and MC_UU_12025. We thank Fatma Kok and Douglas Vernimmen for critical reading of the manuscript. This research was supported by the British Heart Foundation (BHF Oxford CoRE and BHF IBSR Fellowship FS/13/50/30436 to R.M. and M. K.), by a Wellcome Trust Chromosome and Developmental Biology PhD Scholarship (\#WT102345/Z/13/Z. to T.D.) and by the MRC MHU programme number MC_UU_12009/8.

\section{Data Availability}

All data generated or analysed during this study are included in this published article (and its supplementary information files). Accession codes for ATACseq data will be available before publication.

\section{Author contributions}

T.D., M.K., C.K., E.P. and R.M. designed the study. T.D., M.K., C.K., J.P-Z., K.G.,C.M. and R.M. performed experiments and analyzed the data. J.P-Z., B.F. and K.G. performed experiments. R.R. performed the bioinformatics analyses, T.D. and R.M. wrote the paper and R.P., E.P. and R.M. edited the paper. R.P., E.P. and R.M. secured funding. 
bioRxiv preprint doi: https://doi.org/10.1101/516203; this version posted December 10, 2019. The copyright holder for this preprint (which was not certified by peer review) is the author/funder, who has granted bioRxiv a license to display the preprint in perpetuity. It is made available under aCC-BY-NC-ND 4.0 International license.

\section{Declaration of interests}

The authors declare no competing interests. 
bioRxiv preprint doi: https://doi.org/10.1101/516203; this version posted December 10, 2019. The copyright holder for this preprint (which was not certified by peer review) is the author/funder, who has granted bioRxiv a license to display the preprint in perpetuity. It is made available under aCC-BY-NC-ND 4.0 International license.

\section{Figures and Figure legends}


bioRxiv preprint doi: https://doi.org/10.1101/516203; this version posted December 10, 2019. The copyright holder for this preprint (which was not certified by peer review) is the author/funder, who has granted bioRxiv a license to display the preprint in perpetuity. It is made available under aCC-BY-NC-ND 4.0 International license.
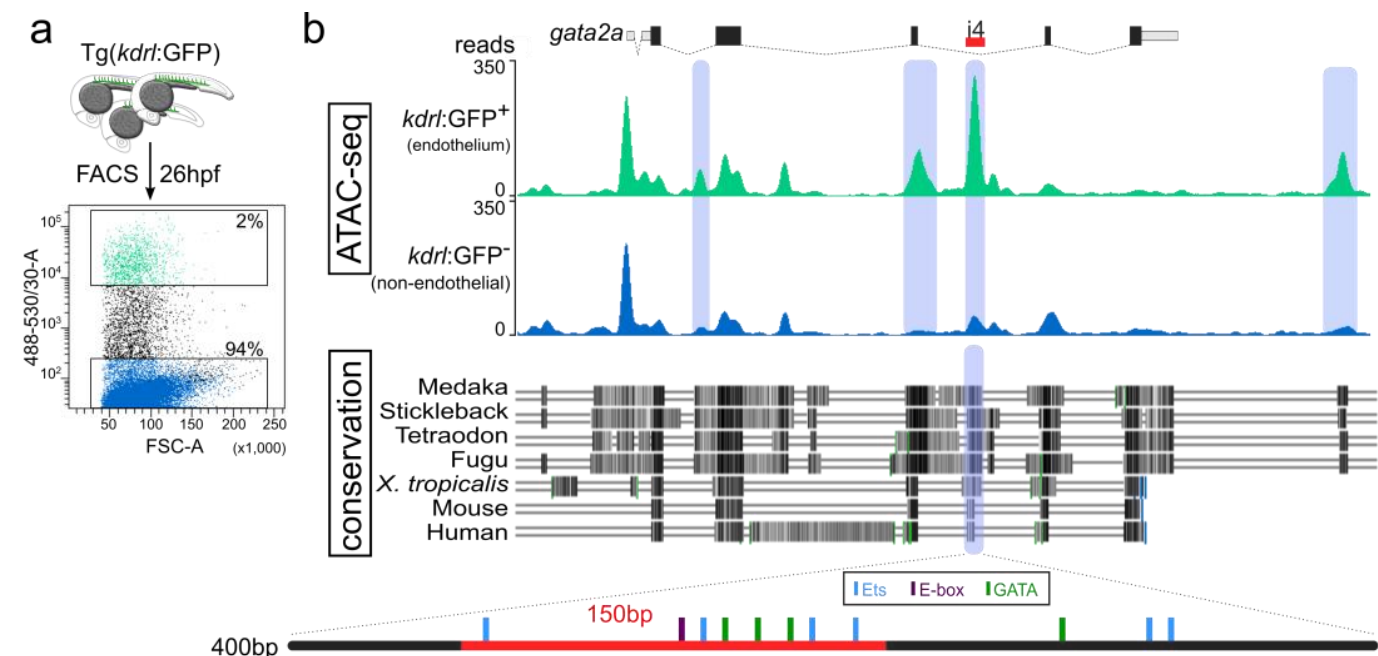

C

Human

Chicken

Mouse

Opossum

Xenopus

Zebrafish

Human

Chicken

Mouse

Opossum

Xenopus

Zebrafish

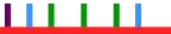

\begin{abstract}
-TATTATTTTTCCATGGAGTCAC----CTATACTGTGTATTTTCATTTGAGTGATTTT--AAAAAAATGCCCTTTCGGATCTCCTGC --TATTATTTTCCATGGAGTCAC----CTATACTTTGTATTTTCATTCGAGCGATTTA--AAAAAAATGTCCTTTCTGAGCTCCTGG -TATTATTTTTCCATGGAGTCAC----CTATACTGTGTATTTTCATTTGAGTGATTTTTTAAAAAAATGTCCTTTCGGATCTCCTGC TATTATTTTTTCCATGGAGTCAC----CTATACTGTGTATTTTCATTTGAGTGATTTTT--TTTAAATGCCCTTTCTAAGCTCCTGG --ATTCATTTTCCATGGAGTCAC----CTACACTCTGTACTTTGGGGCCTTTTCTTTGT-TGTAAGTGGCCCCATGTGAGATCATGC

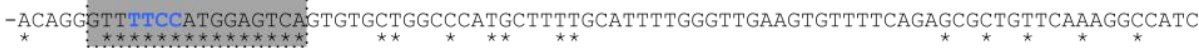

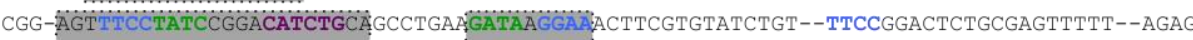
TAGTAGTTTCCTATCCGGATATCTGTACGTTAAAGATAAGGAAACTTTGTGTATCTGT--TTCCTGACTCTAAAAGCCTTAG-AGA CGG-ÄGTTTCCTATCCGGACATCTGCÄGCCGGTAGATAAGGAAACTTCGTGTATCTGT--TTCCGGAC-CGGCAAGTTTTC--AGAG CCAGAGTTTCCTATCCGGACATCTGCÄGGCTGAR:GATAAGGAAACTTAGTGTATCTGT--ATCCGGACTCTGCGAGCTTTGAGAGTG TAGTGGCTTCCTATCCGGATATGTGCATTGTGCT:GATAAGGAACTTTGTGTCTCTGT--TTCCGGACATTAAATAACTTTAGAGAG CAGTTGTTTCCTATCCGGATATCTGCCCGGCCCGATAAGGAAGCCAGGCTCCCGGGTGTTTCCGTCTTCAACAAACCCGGCTGAAT
\end{abstract}

gata2a i4-1.1kb:EGFP

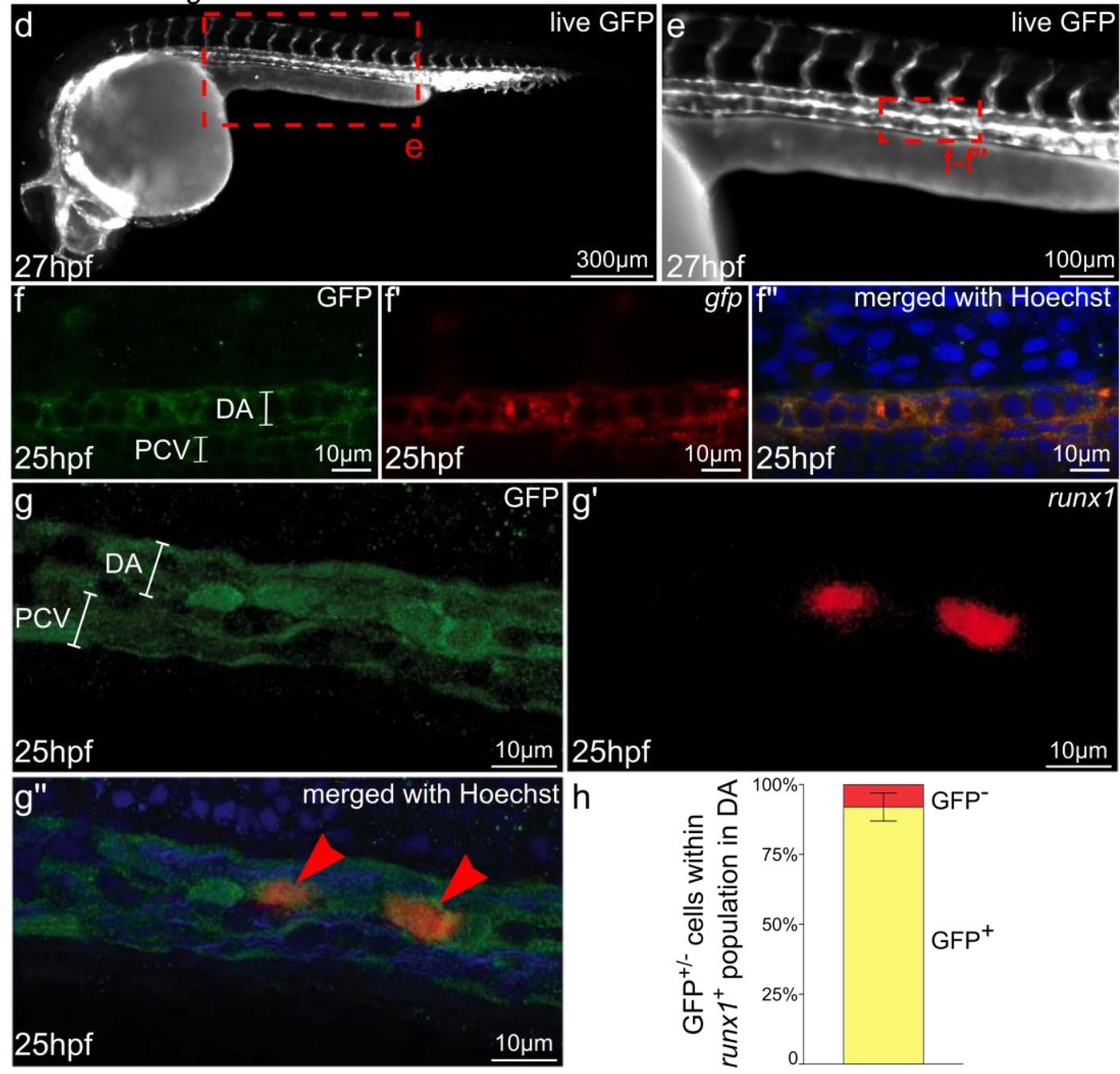


Figure 1. The i4 enhancer in the gata2a locus is conserved and drives pan-endothelial expression of a GFP reporter in zebrafish. (a) Kdrl:GFP ${ }^{+}$(green) and $k d r l: G F P^{-}$(blue) cells were FACS-sorted from $26 \mathrm{hpf}$ embryos and used for preparation of ATAC-seq libraries. (b) The image of the mapped reads represents stacked means of two biological ATAC-seq replicates. Differential peak analysis identified four chromatin regions (blue shading) in the locus of gata2a that are significantly more open in the $k d r l: \mathrm{GFP}^{+}$population $(p<0.0001)$. A region in the fourth intron (termed i4 enhancer) is conserved throughout vertebrates. Black and grey shading denotes regions of high conservation between the species analysed (c) The highly conserved 150bp region (red) contains putative transcription factor binding sites, mapped computationally. Light blue: Ets binding sites; purple: E-box binding sites; green: GATA binding sites; asterisks: conserved residues. (d) Widefield fluorescent image of a live Tg(gata2a-i4-1.1kb:GFP) zebrafish embryo at 27hpf showing GFP fluorescence in the endothelial cells and in the heart (endocardium). (e) Higher magnification image of the trunk of the embryo from panel d. $\left(f-f^{\prime \prime}\right)$ Confocal images of a trunk fragment of a $\operatorname{Tg}($ gata2ai4-1.1kb:GFP) embryo immunostained with anti-GFP antibody (f) and probed for $g f p$ mRNA ( $\left.f^{\prime}\right)$ at 25hpf. ( $\left.f^{\prime \prime}\right)$ Merged images from panels f-f' with Hoechst nuclear staining in blue, showing complete overlap of GFP protein and mRNA. (g-g") Confocal images of the dorsal aorta (DA) and posterior cardinal vein (PCV) of a $\operatorname{Tg}$ (gata2a-i4-1.1kb:GFP) embryo immunostained with anti-GFP antibody (g) and probed for runx1 mRNA $\left(g^{\prime}\right)$ at $25 \mathrm{hpf}$. See panel e for approximate position within the embryo. ( $\left.\mathrm{g}^{\prime \prime}\right)$ Merged images from panels g-g', also showing Hoechst nuclear staining in blue. (h) Counting of the run $\times 1^{+}$cells represented in panels $g^{\prime}-g^{\prime \prime}$ in 25 embryos shows that $>90 \%$ of $r u n \times 1^{+}$cells are also GFP+ $. N=3$. Error bars: \pm SD. See also Supplementary Figure 1. 


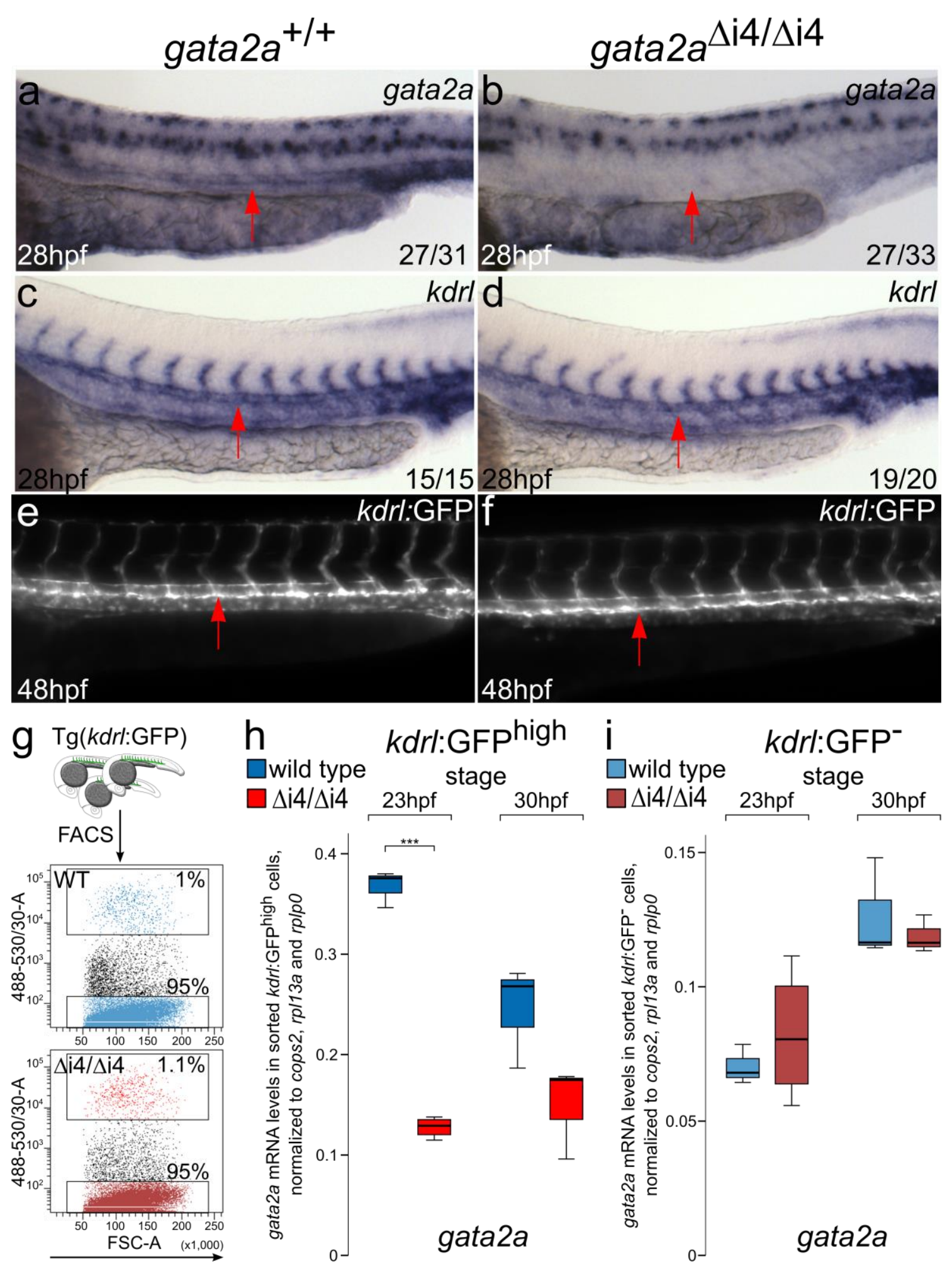

Figure 2. Deletion of the i4 enhancer in gata2 $a^{\Delta \mathrm{i} 4 / \Delta \mathrm{i} 4}$ mutants leads to reduced levels of gata2a mRNA in the endothelium. (a-b) A significant majority of gata $2 a^{\Delta \mathrm{i} 4 / \Delta \mathrm{i} 4}$ mutants have reduced levels of gata2a mRNA in the dorsal aorta (arrows) at 28hpf, compared to wild type siblings, as detected with in situ hybridization. $\left(X^{2}=10.720\right.$, d.f. $\left.=1, p<0.01\right) * * p<0.01$. The expression in the neural tube appears unaffected. (c-d) In situ 
hybridization for the endothelial marker $k d r l$ at $28 \mathrm{hpf}$ reveals no difference between $g a t a 2 a^{\Delta i 4 / \Delta i 4}$ mutants and wild type siblings. The dorsal aorta (arrows) appears unaffected. (e-f) Live images of the trunks of $48 \mathrm{hpf}$ $\operatorname{Tg}(k d r l: G F P)$ and $\operatorname{Tg}(k d r l: G F P) ; g a t a 2 a^{\Delta i 4 / \Delta i 4}$ embryos show normal vascular morphology in the mutants. The endothelium of the dorsal aorta (arrows) appears normal in the gata2 $2 a^{\Delta i 4 / \Delta i 4}$ embryos. (g) Kdrl:GFPhigh and $k d r l:$ GFP- $^{-}$cells were sorted from non-mutant (WT, blue) and gata2 $a^{\Delta \mathrm{i} / / \Delta 4}$ (red) embryos carrying the $\mathrm{Tg}(k d r l: G F P)$ transgene. (h-i) qRT-PCR on RNA isolated from the sorted $k d r l: G F P^{\text {high }}$ or $k d r l:$ GFP $^{-}$cells (panel g) shows decreased levels of gata2a mRNA in the endothelium of gata2 $2 a^{\Delta i 4 / \Delta i 4}$ mutants at $23 \mathrm{hpf}$ $(t=20.026$, d.f. $=5, p<0.001)$ compared to wild type. At 30hpf this difference is not statistically significant $(t=2.146, d . f .=4, p=0.098)$. There is no difference in gata $a$ mRNA levels in non-endothelial cells between wild type and gata2a $a^{\Delta i / \Delta i 4}$ mutants (23hpf: $t=0.69$, d.f. $=5, p>0.5 ; 30 h p f: t=0.618$, d.f. $=4, p>0.5$ ). $N=4$ for gata $2 a^{\Delta i 4 / \Delta i 4}$ at $23 \mathrm{hpf}, \mathrm{N}=3$ for other samples. Note different scales of expression levels. ${ }^{* * *} p<0.001$. See also Supplementary Figure 2. 


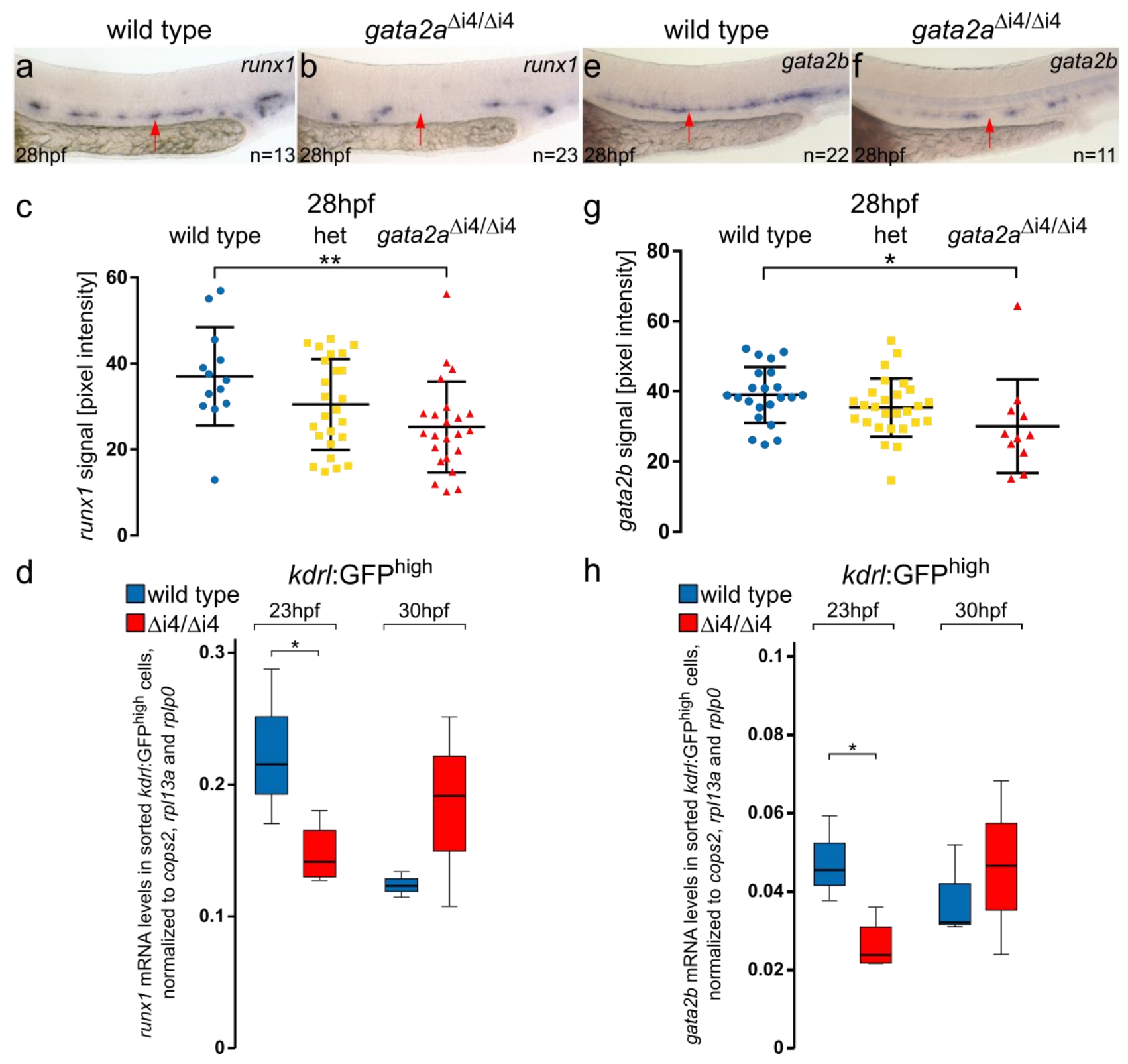

Figure 3. Loss of gata2a expression in the endothelium of gata2a $a^{\Delta i 4 / \Delta i 4}$ mutants leads to decreased levels

of runx 1 and gata $2 b$ in the HE. (a-b) In situ hybridization for runx1 expression in the HE (arrows) of wild type and gata2 $a^{\Delta \mathrm{\Delta i} / \Delta \mathrm{\Delta i}}$ embryos at 28hpf. (c) Quantification of the runx1 in situ hybridization signal from wild type (blue), heterozygous gata2 $a^{+/ \Delta i 4}$ (het, yellow) and gata2a $a^{\Delta i / \Delta i 4}$ (red) siblings at $28 \mathrm{hpf}$ shows significant decrease in runx1 pixel intensity in the DA in the homozygous mutants compared to wild type $\left(\mu_{\mathrm{wt}}=34.8\right.$, $\mu_{\text {mut }}=25.3 ; F=4.956$, d.f. $=2,58 ;$ ANOVA) ${ }^{* *} p<0.01 . n=14$, wild type; $n=25$, het; $n=23$, gata $2 a^{\Delta i 4 / \Delta i 4}$. Error bars: mean $\pm S D$. (d) qRT-PCR on RNA isolated from the sorted $k d r l: G F P^{+}$cells shows decreased levels of $r u n x 1$ 
mRNA in the endothelium of $g a t a 2 a^{\Delta i 4 / \Delta i 4}$ mutants at $23 \mathrm{hpf}(t=2.585$, d.f. $=5, p<0.05)$ but not at $30 \mathrm{hpf}$ $(t=1.326, d . f .=4, p>0.2)$, compared to wild type. $N=4$ for gata2 $a^{\Delta i / / \Delta i 4}$ at $23 \mathrm{hpf}, \mathrm{N}=3$ for other samples. Note different scales of expression levels. ${ }^{*} p<0.05$. (e-f) Gata $2 b$ expression in the HE (arrows) of wild type and gata2a $a^{\Delta i 4 / \Delta i 4}$ embryos at 28hpf. (g) Quantification of the gata2b mRNA signal, detected by in situ hybridization, from wild type (blue), heterozygous gata2a $a^{+/ \Delta i 4}$ (het; yellow) and gata2a $a^{\Delta i / \Delta i 4}$ (red) siblings at 28hpf shows significant decrease in gata $2 b$ pixel intensity in the DA in the homozygous mutants compared to wild type $\left(\mu_{\mathrm{wt}}=39, \mu_{\mathrm{mut}}=30.1 ; F=5.05\right.$, d.f. $=2,54 ;$ ANOVA), ${ }^{*} p<0.05 . \mathrm{n}=22$, wild type; $\mathrm{n}=24$, het; $\mathrm{n}=11$, gata2a $a^{\Delta i / / \Delta i 4}$. Error bars: mean \pm SD. (h) qRT-PCR in sorted $k d r l: \mathrm{GFP}^{+}$cells showed decreased levels of gata2b mRNA in the endothelium of $g a t a 2 a^{\Delta i 4 / \Delta i 4}$ mutants at $23 \mathrm{hpf}(t=3.334$, d.f. $=5, p<0.05)$ but not at $30 \mathrm{hpf}$ $\left(t=0.373\right.$, d.f. $=4, p>0.7$ ), compared to wild type. $\mathrm{N}=4$ for gata2 $a^{\Delta \mathrm{i} / / \Delta \mathrm{i4}}$ at $23 \mathrm{hpf}, \mathrm{N}=3$ for other samples. ${ }^{*} p<0.05$. See also Supplementary Figures 3 and 4. 

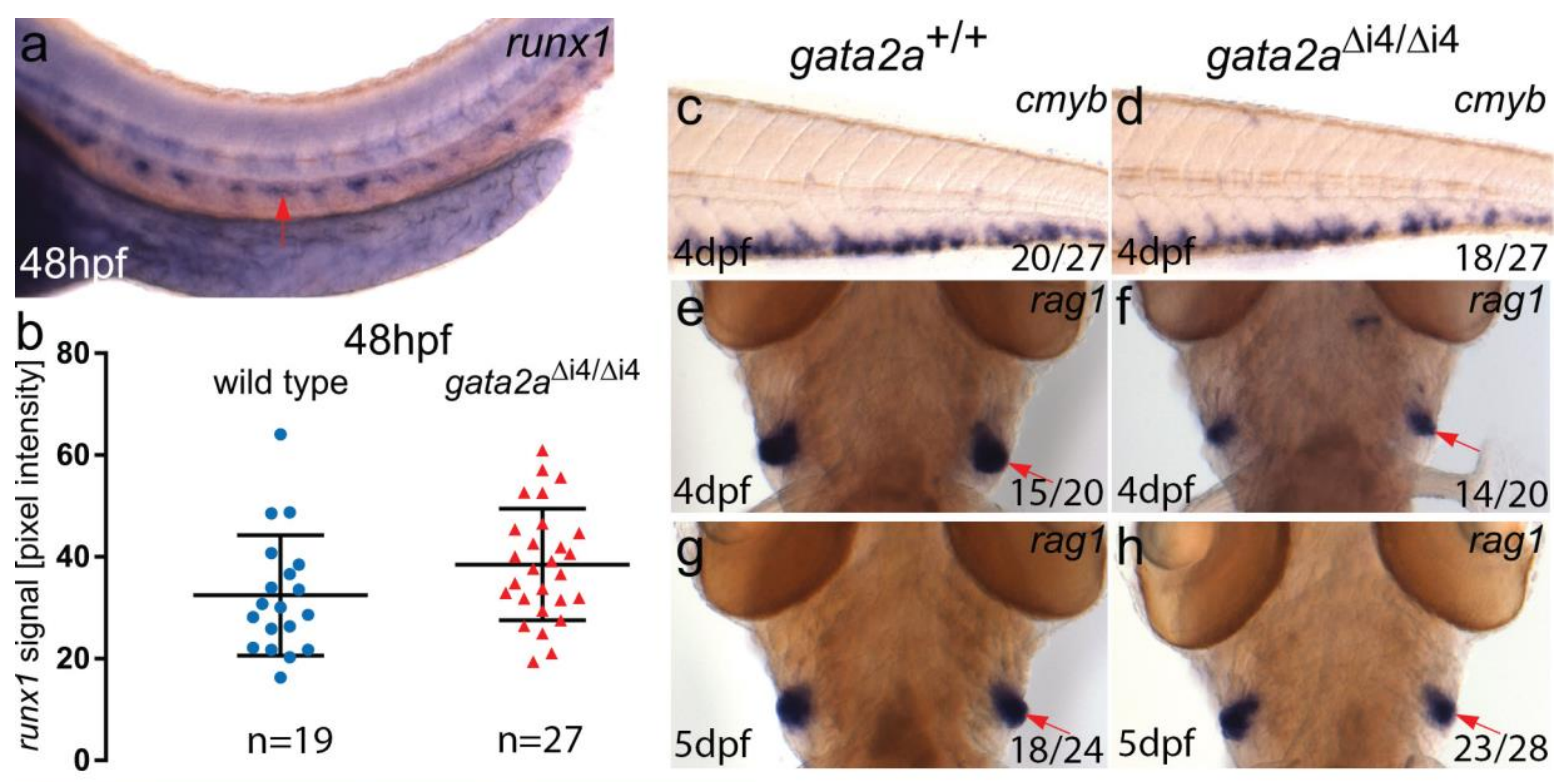

rag1
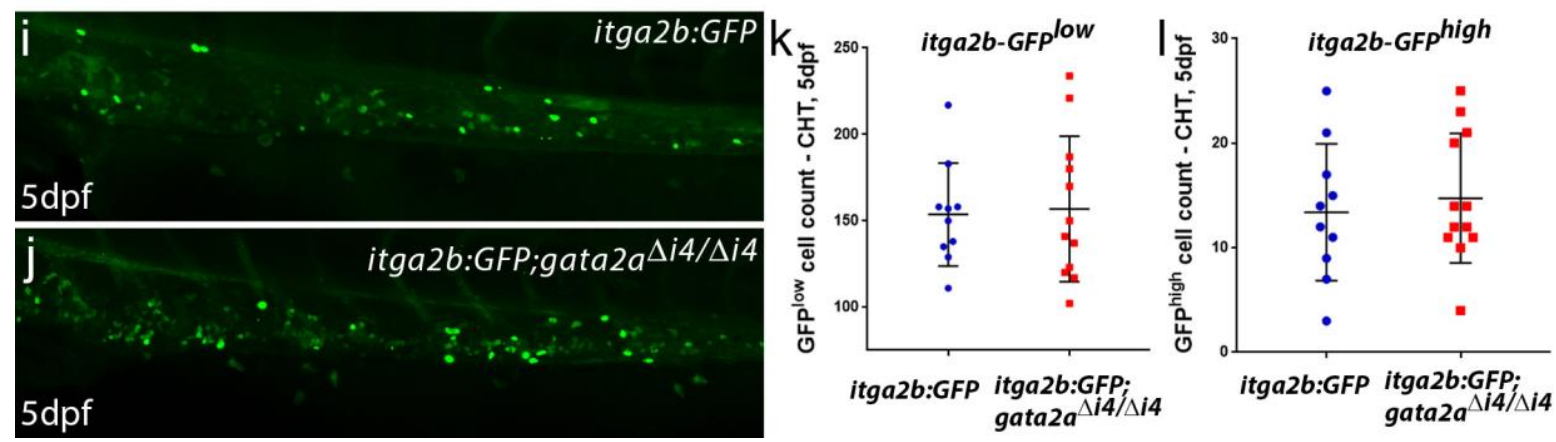

Figure 4. Gata2a $a^{\Delta i / \Delta i 4}$ mutants display a recovery of the initial haematopoietic defects from $48 \mathrm{hpf}$. (a)

Representative image of runx1 expression in the trunk of a wild type embryo at 48hpf showing runx1 mRNA in the dorsal aorta (arrow). (b) Quantification of the runx1 in situ hybridization signal in wild type (blue) and gata $2 a^{\Delta i / / \Delta i 4}$ mutants (red) siblings at $48 \mathrm{hpf}$. There is no significant difference in runx1 pixel intensity in the DA between the homozygous mutants and wild type $\left(\mu_{\mathrm{wt}}=33.1, \mu_{\mathrm{mut}}=37.5, t=1.410, \mathrm{~d} . \mathrm{f}=44, \mathrm{p}=0.17 . \mathrm{n}=19\right.$, wild type; $\mathrm{n}=27$, gata2 $a^{\Delta i 4 / \Delta i 4}$ ). Error bars: mean \pm SD. (c-d) In situ hybridization for $c m y b$ in the CHT. We detected no difference in expression between wild type and gata2a $a^{\Delta i / / \Delta i 4}$ siblings at $4 \mathrm{dpf}$. (e-h) In situ hybridization (ventral image) for rag1 in the thymii, showing a slight decrease (relative to wild type) in rag1 (red arrows) in approximately half of the homozygous mutant embryos at $4 \mathrm{dpf}$. This effect is absent at 5dpf. (i-j) Maximum projections of itga2b:GFP transgenic embryos in the CHT at $5 \mathrm{dpf}$ in (i) wild type and (j) gata2 $a^{\Delta i 4 / \Delta i 4}$ siblings. (k) HSPC (itga2b:GFPlow) counts in the CHT of wild type $(n=10)$ and gata $2 a^{\Delta i 4 / \Delta i 4}$ mutants ( $n=12)$ at $5 \mathrm{dpf}$. No difference was detected between genotypes $\left(\mu_{\mathrm{wt}}=153.5 ; \mu_{\mathrm{mut}}=145.5 ; p=0.98\right.$, 
bioRxiv preprint doi: https://doi.org/10.1101/516203; this version posted December 10, 2019. The copyright holder for this preprint (which was not certified by peer review) is the author/funder, who has granted bioRxiv a license to display the preprint in perpetuity. It is made available under aCC-BY-NC-ND 4.0 International license.

Mann-Whitney test). (I) Thrombocyte (itga2b:GFP ${ }^{\text {high }}$ ) counts in the CHT of wild type $(\mathrm{n}=10)$ and gata2a $a^{\Delta i / \Delta i 4}$ mutants $(n=12)$ at $5 \mathrm{dpf}$. No difference was detected between genotypes $\left(\mu_{\mathrm{wt}}=13 ; \mu_{\mathrm{mut}}=13 ; p=0.71\right.$, MannWhitney test). The scatter plots show the median \pm SD. 
a

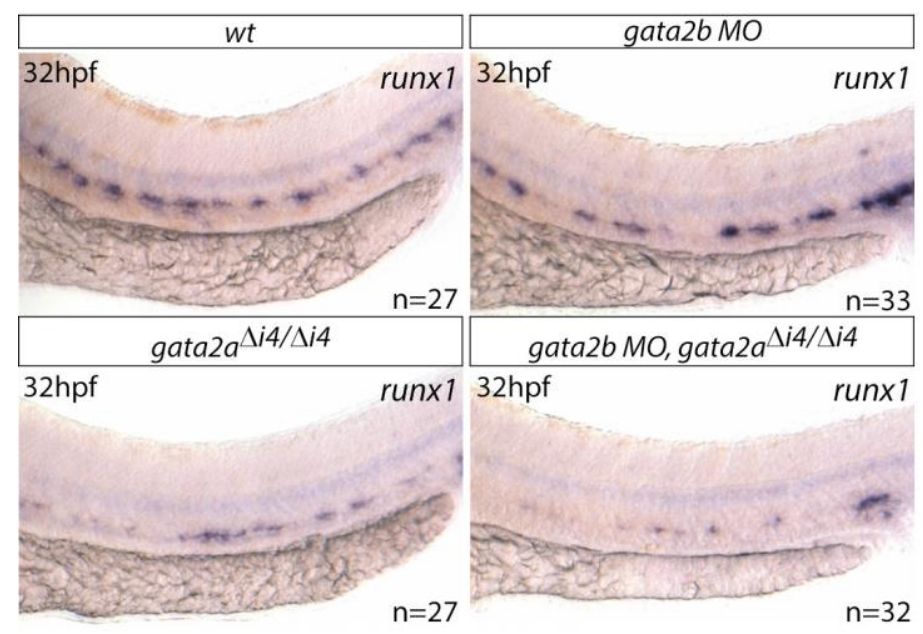

C

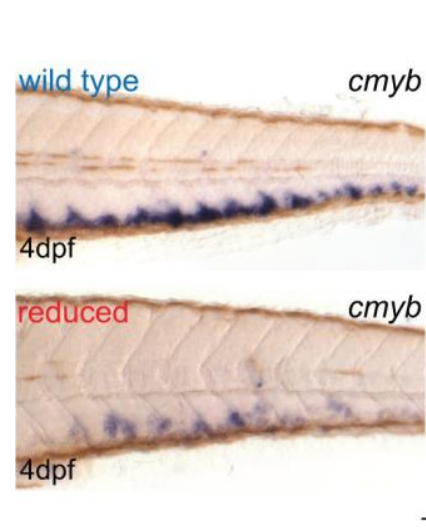

$\mathrm{e}$

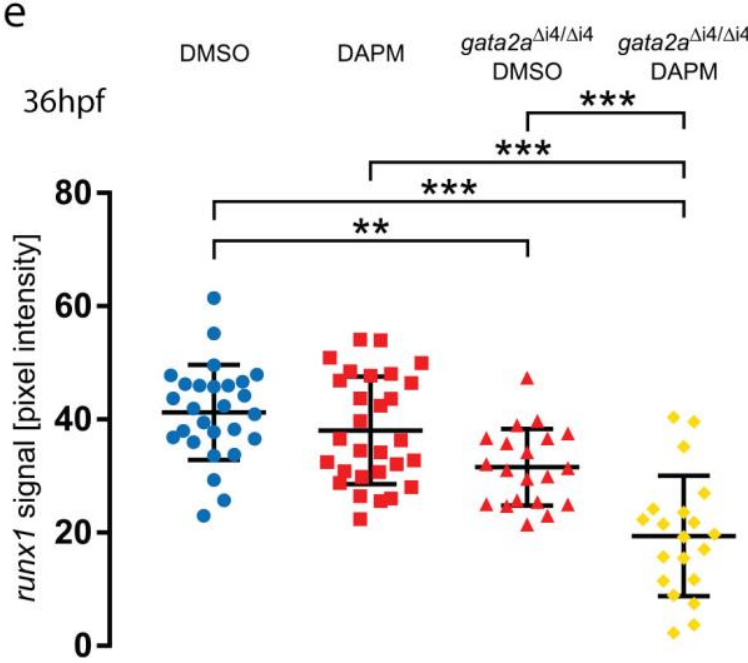

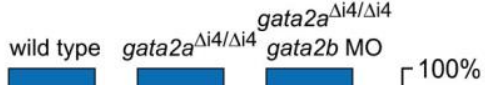

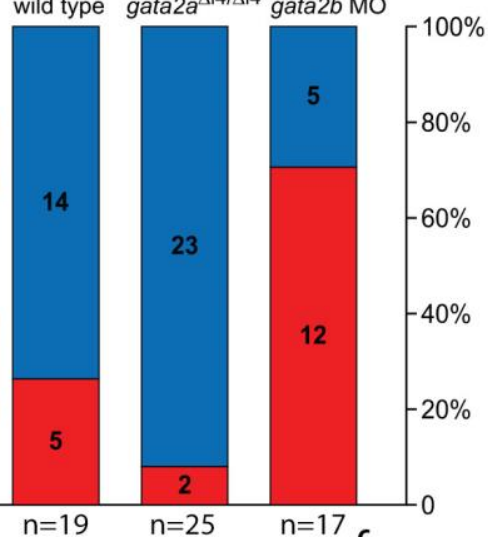
f b $32 \mathrm{hpf}$ wild type gata2b MO gata2a $a^{\mathrm{\Delta i} / / \Delta \mathrm{i} 4}$ gata2a $2 a^{\mathrm{\Delta i} / / \Delta \mathrm{i} 4}$
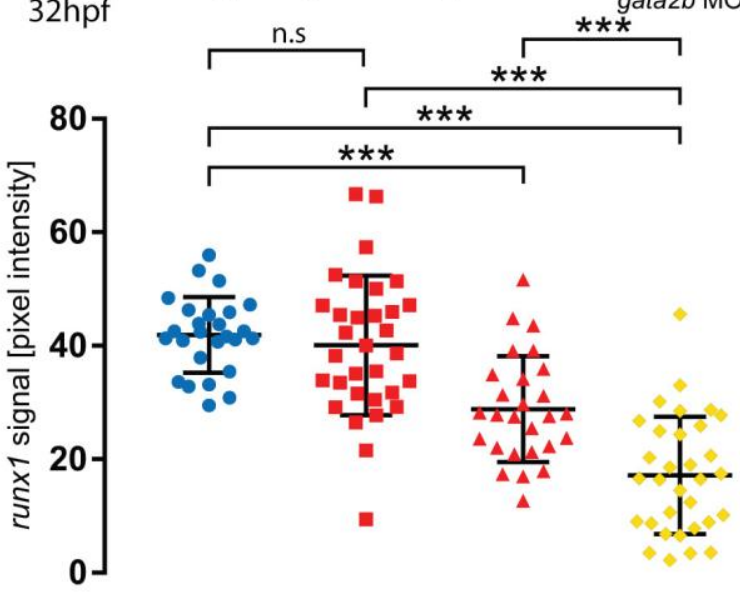

d $28 \mathrm{hpf}$ wild type gata2a $^{\Delta \mathrm{i} 4 / \Delta \mathrm{i} 4} \quad \begin{aligned} & \text { wild type } \\ & \mathrm{i} 4-\text { gata2b }\end{aligned}$

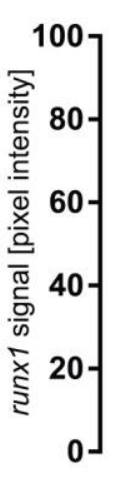

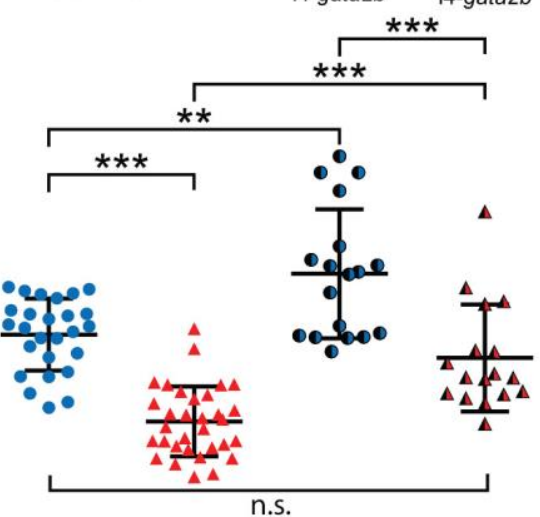
$w t$ $\begin{array}{llr}\text { runx1 } & \text { wt }+25 \mu \mathrm{hpf} & \text { runx } 1\end{array}$
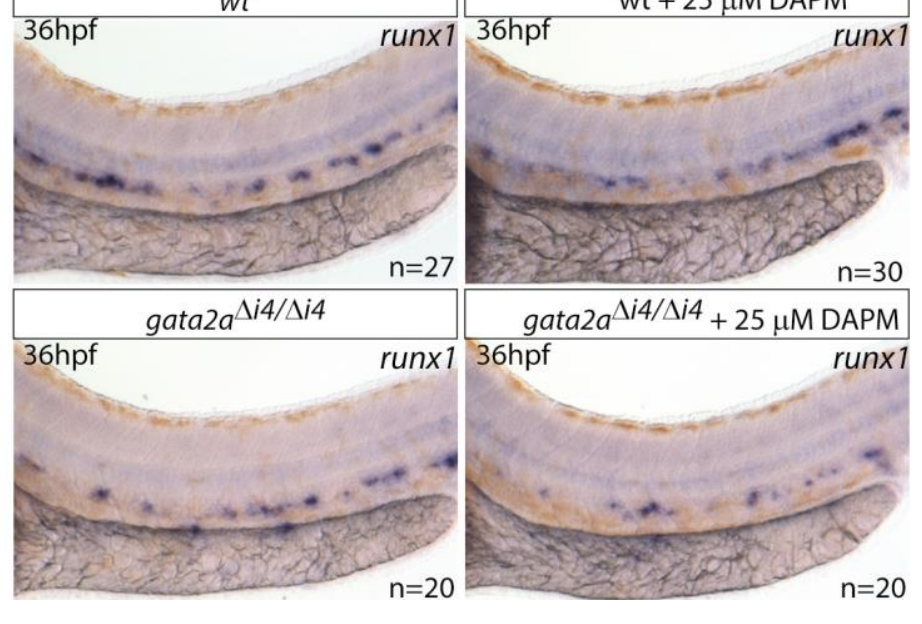

Figure 5. Gata2b and Notch signalling are sufficient to recover haematopoietic markers in gata2 $a^{\Delta i 4 / \Delta i 4}$

mutants. (a) Expression of runx1 in HE at $32 \mathrm{hpf}$ in wild type (wt), gata2b MO-injected (7.5ng) wt embryos, gata2 $a^{\Delta i 4 / \Delta i 4}$ mutants and gata $2 b \mathrm{MO}$-injected (7.5ng) gata2a $a^{\Delta \mathrm{i} / / \Delta \mathrm{i} 4}$ mutants. (b) Quantification of the runx1 in situ hybridization (ISH) signal in wt, gata $2 b$ morphants, gata $2 a^{\Delta i 4 / \Delta i 4}$ mutants and gata $2 a^{\Delta i 4 / \Delta i 4}$ mutants 
injected with gata2b MO. runx1 expression is decreased in gata $2 a^{\Delta \mathrm{ii} / \Delta \mathrm{it}}$ mutants $\left(\mu_{\mathrm{wt}}=41.9, \mu_{\mathrm{mut}}=28.9\right.$; $F=44.641$, d.f. $=3,62.3 ; p<0.001)$. Gata2b MO knockdown significantly decreases runx 1 in the DA of gata $2 a^{\Delta i 4 / \Delta i 4}$ mutants $\left(\mu_{\mathrm{mut}}=28.9, \mu_{\mathrm{mut}+\mathrm{MO}}=17.2\right)$, but not wt embryos at $32 \mathrm{hpf}\left(\mu_{\mathrm{wt}}=41.9, \mu_{\mathrm{MO}}=40.1 ; \mathrm{p}=0.89\right.$, $\mathrm{p}=0.89$, Games-Howell post-hoc test, Welch's ANOVA). $n=27, w t ; n=27$, gata2a $a^{\Delta \mathrm{i} 4 / \Delta \mathrm{i} 4} ; \mathrm{n}=33, \mathrm{wt}+$ gata2b MO; $\mathrm{n}=32$, gata2a $a^{\Delta \mathrm{i} / / \Delta \mathrm{i} 4}+$ gata2b MO. (c) Scoring cmyb expression at $4 \mathrm{dpf}$ in wt, gata2a $a^{\Delta i / / \Delta i 4}$ mutants and gata2a $a^{\Delta \mathrm{i} / / \Delta \mathrm{i} 4}$ mutant embryos injected with gata2b MO as wt (blue) or reduced (red). Gata2b MO knockdown (7.5ng) inhibits the haematopoietic recovery of gata $2 a^{\Delta i 4 / \Delta i 4}$ mutants. $\left(X^{2}=18.784\right.$, d.f. $=2, p<0.001$ ). (d) Quantification of the runx1 ISH signal, from $28 \mathrm{hpf}$ wt embryos (blue), gata2a $a^{\Delta i / / \Delta i 4}$ mutants (red) and their siblings injected with a gata2a-i4-450bp:gata2b construct (shaded blue and red). Ectopic expression of gata $2 b$ increases runx 1 expression in the HE of wt embryos $\left(\mu_{\mathrm{wt}}=38.8, \mu_{\mathrm{wt}+\text { gata2 } b}=53.4\right.$; $p<0.01)$ and rescues runx1 expression in the DA of $g a t a 2 a^{\Delta i 4 / \Delta i 4}$ mutants to wt levels $\left(\mu_{\text {mut }}=17.9\right.$, $\mu_{\text {mut }+ \text { gata } 2 b}=33.2 ; p<0.001 ; \mu_{\mathrm{wt}}=38.8, \mu_{\text {mut+gata2b }}=33.2 ; \mathrm{p}=0.31$, Tukey HSD post-hoc test). $\mathrm{n}=25, \mathrm{wt} ; \mathrm{n}=33$, gata2a $a^{\Delta i 4 / \Delta i 4} ; \mathrm{n}=18, \mathrm{wt}+$ gata2a-i4-450bp:gata2b construct; $\mathrm{n}=17$, gata2a $a^{\Delta \mathrm{i} / / \mathrm{i} 4}+$ gata2a-i4-450bp:gata2b construct. (e) Quantification of the runx1 ISH signal at 36hpf in embryos treated with a suboptimal dose $(25 \mu \mathrm{M})$ of the Notch inhibitor DAPM. $25 \mu \mathrm{M}$ DAPM showed no effect on runx1 expression in wt compared to DMSO-treated embryos ( $\mu_{\mathrm{DMSO}}=40.5, \mu_{\mathrm{DAPM}}=38 ; \mathrm{p}=0.735$, Tukey HSD post-hoc test). DMSO-treated gata2 $a^{\Delta \mathrm{i} / \Delta \mathrm{\Delta i}}$ mutants show a decrease in runx1 expression $\left(\mu_{\mathrm{DMSO}}=40.5, \mu_{\text {mut+DMso }}=31.5 ; F=25.774\right.$, d.f. $=3,91$; ANOVA). DAPM treatment significantly reduced runx1 expression in the DA gata2a $a^{\Delta i / / \Delta i 4}$ mutants $\left(\mu_{\text {mut+DMSO }}=31.5, \mu_{\text {mut+DAPM }}=19.4\right) . n=27, w t+D M S O ; n=20, g a t a 2 a^{\Delta i 4 / \Delta i 4}+D M S O ; n=30, w t+D A P M ; n=20$, gata2 $a^{\Delta i / / \Delta i 4}+$ DAPM. (f) Representative images of the average runx1 expression at $36 \mathrm{hpf}$ in wt and gata2 $a^{\Delta \mathrm{i} / / \Delta \mathrm{i} 4}$ mutants treated with $25 \mu \mathrm{M}$ DAPM. Error bars: mean \pm SD. ${ }^{* *} p<0.01 ;{ }^{* * *} p<0.001$. See also Supplementary Fig. 5. 


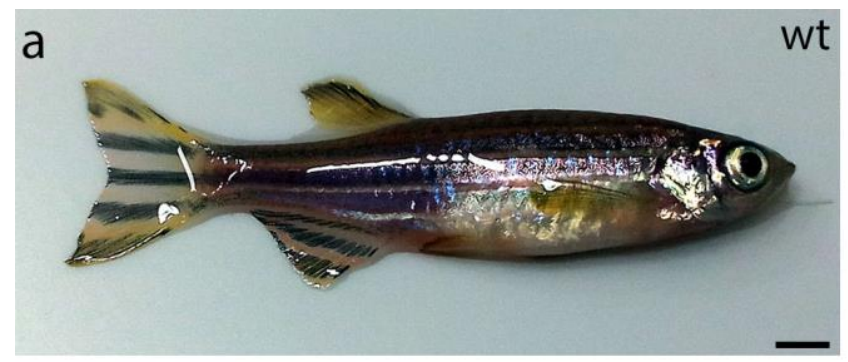

C

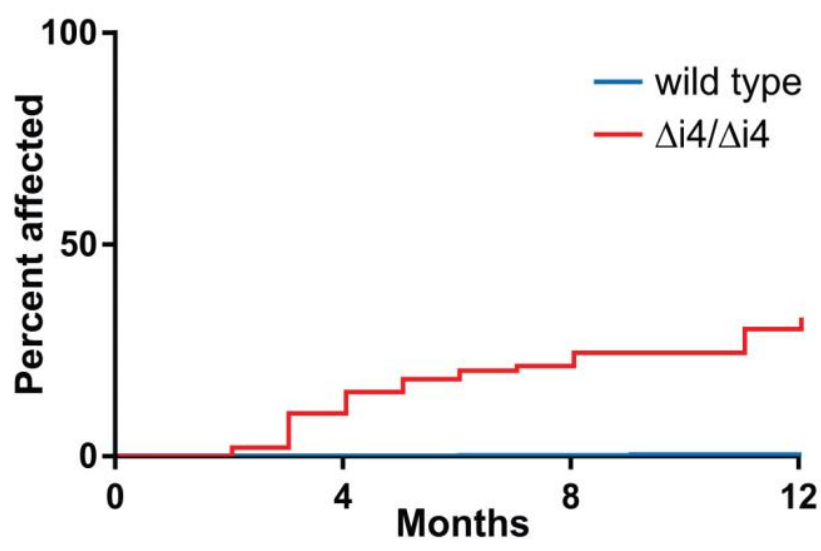

$f$

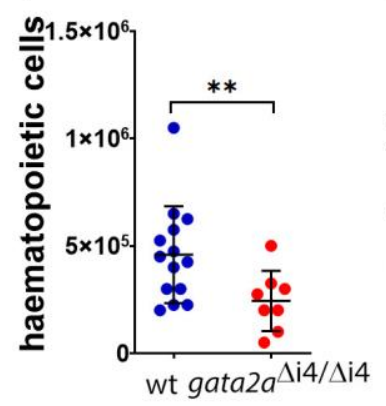

g

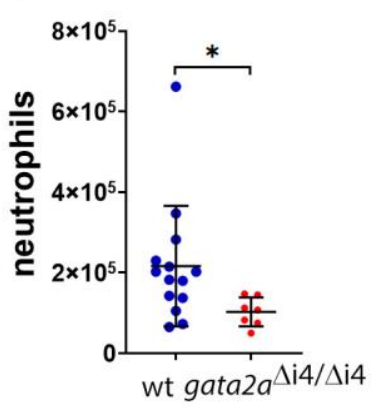

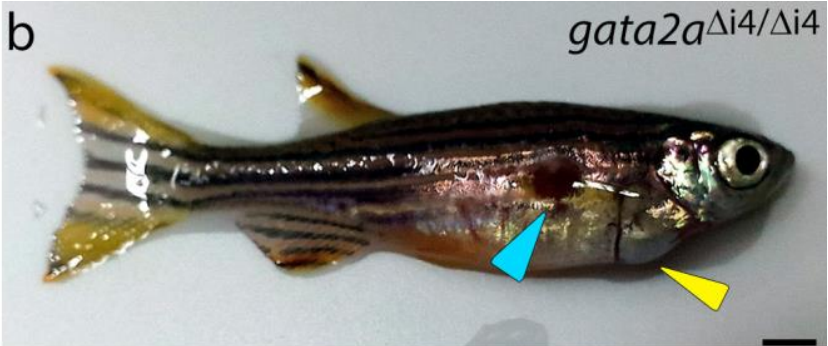

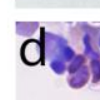

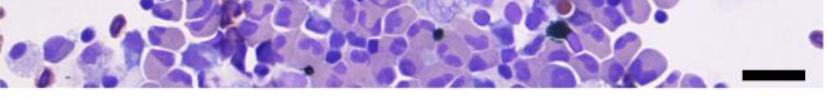
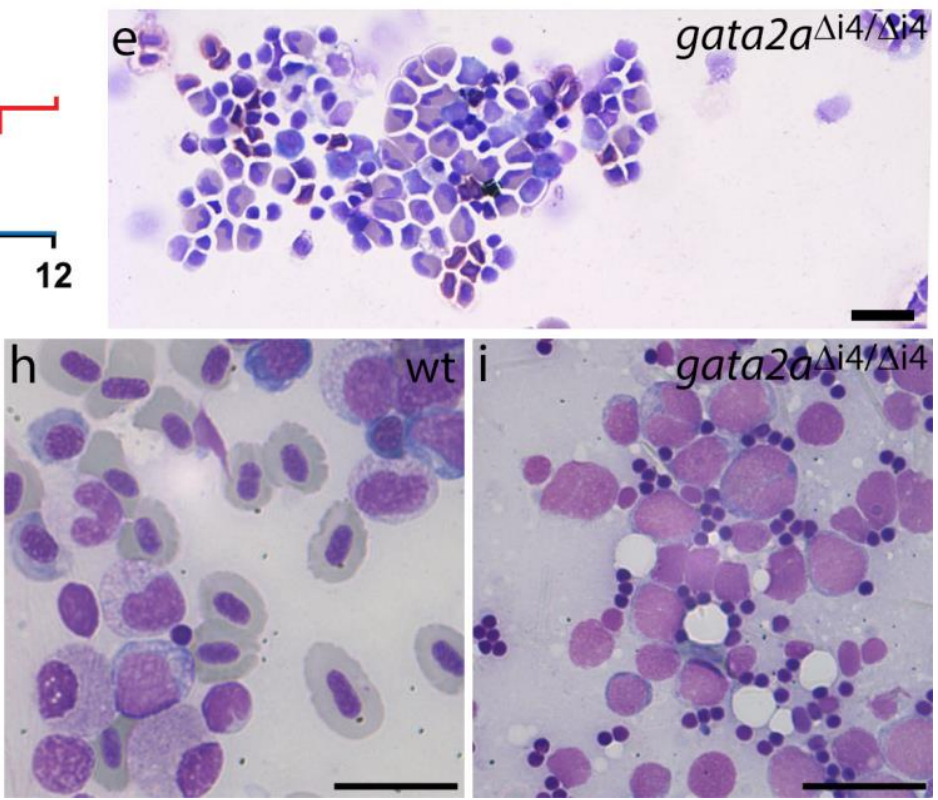

Figure 6. Gata2 $a^{\Delta \mathrm{i} / \Delta \mathrm{\Delta i} 4}$ mutants show cardiac oedema, hypocellularity and marrow failure. (a-b) General

morphology of zebrafish adults: (a) wild type; (b) gata2a $a^{\Delta / 4 / \Delta i 4}$ mutant showing skin infection (blue

arrowhead) and pericardial oedema (yellow arrowhead). (c) Over $25 \%$ ( $n=29 / 108)$ of gata2 $a^{\Delta i 4 / \Delta i 4}$ mutants

(red) catch infections or suffer from heart oedemas by 6 months. Only around $65 \%$ ( $n=69 / 108)$ survive for

more than 12 months without overt signs of infections. Fewer than $1 \%(n=2 / 500)$ of wild type fish (blue)

exhibit such defects. The graph does not include deaths by other causes. (d-e) May-Grunwald/Wright-

Giemsa staining in cytospins of haematopoietic cells isolated from the WKM of zebrafish adults: (d) wild type; (e) gata2 $a^{\mathrm{\Delta i} / / \Delta \mathrm{i} 4}$ mutant. Note the decrease in cell numbers. (f) Cell counts of haematopoietic cells isolated from WKM of wild type $(n=14)$ and gata $2 a^{\Delta i 4 / \Delta i 4}$ mutants $(n=8)$. The gata2 $2 a^{\Delta i 4 / \Delta i 4}$ mutants show a 
$\sim 2$-fold decrease in haematopoietic cell numbers in the WKM $\left(\mu_{\mathrm{wt}}=4.37 \times 10^{5} ; \mu_{\mathrm{mut}}=2.37 \times 10^{5}, p=0.0185\right.$, Mann-Whitney test). (g) Number of neutrophils isolated from WKM of wild type $(n=14)$ and gata2a $a^{\Delta i 4 / \Delta i 4}$ mutants $(n=7)$. The gata2a $a^{\Delta i 4 / \Delta i 4}$ mutants show a 2 -fold decrease in neutrophil numbers in the WKM $\left(\mu_{\mathrm{wt}}=2.17 \times 10^{5} ; \mu_{\mathrm{mut}}=1.03 \times 10^{5}, p=0.0269\right.$, Mann-Whitney test). The scatter plots show the median cell number $\pm S D$. (h-i) Kidney smears from 9 months post-fertilization adult animals were assessed. (h) Wild type shows various stages of lineage differentiation. (i) WKM smear; 1 of 10 gata2 $a^{\Delta i 4 / \Delta i 4}$ mutants showed the presence of excess blasts with very little erythroid differentiation (98\% blasts, $>200$ cells assessed). Scalebars: $2 \mathrm{~mm}(\mathrm{a}, \mathrm{b})$ and $10 \mu \mathrm{m}(\mathrm{d}, \mathrm{e}, \mathrm{h}, \mathrm{i})$. See also Supplementary Fig.6. 